\title{
Depth and Stanley depth of the edge ideals of the strong product of some graphs
}

\author{
Zahid Iqbal $^{1 *}$ (D), Muhammad Ishaq ${ }^{1}$ (D), Muhammad Ahsan Binyamin² (D) \\ ${ }^{1}$ School of Natural Sciences, National University of Sciences and Technology, Sector H-12, Islamabad \\ 44000, Pakistan \\ ${ }^{2}$ Department of Mathematics, Government College University Faisalabad, Pakistan
}

\begin{abstract}
In this paper, we study depth and Stanley depth of the edge ideals and quotient rings of the edge ideals, associated with classes of graphs obtained by the strong product of two graphs. We consider the cases when either both graphs are arbitrary paths or one is an arbitrary path and the other is an arbitrary cycle. We give exact formula for values of depth and Stanley depth for some subclasses. We also give some sharp upper bounds for depth and Stanley depth in the general cases.
\end{abstract}

Mathematics Subject Classification (2020). Primary: 13C15, Secondary: 13F20, 05C38, 05E99

Keywords. depth, Stanley depth, Stanley decomposition, monomial ideal, edge ideal, strong product of graphs

\section{Introduction}

Let $S:=K\left[x_{1}, \ldots, x_{n}\right]$ be the polynomial ring over a field $K$. Let $M$ be a finitely generated $\mathbb{Z}^{n}$-graded $S$-module. A Stanley decomposition of $M$ is a presentation of $K$-vector space $M$ as a finite direct sum $\mathcal{D}: M=\bigoplus_{i=1}^{r} w_{i} K\left[A_{i}\right]$, where $w_{i} \in M$ is a homogeneous element in $M, A_{i} \subseteq\left\{x_{1}, \ldots, x_{n}\right\}$ such that $w_{i} K\left[A_{i}\right]$ denote the $K$-subspace of $M$, which is generated by all elements $w_{i} u$, where $u$ is a monomial in $K\left[A_{i}\right]$. The $\mathbb{Z}^{n}$-graded $K$-subspace $w_{i} K\left[A_{i}\right] \subset M$ is called a Stanley space of dimension $\left|A_{i}\right|$, if $w_{i} K\left[A_{i}\right]$ is a free $K\left[A_{i}\right]$-module, where $\left|A_{i}\right|$ denotes the number of indeterminates of $A_{i}$. Define $\operatorname{sdepth}(\mathcal{D})=\min \left\{\left|A_{i}\right|\right.$ : $i=1, \ldots, r\}$, and $\operatorname{sdepth}(M)=\max \{\operatorname{sdepth}(\mathcal{D}): \mathcal{D}$ is a Stanley decomposition of $M\}$. The number $\operatorname{sdepth}(\mathcal{D})$ is called the Stanley depth of decomposition $\mathcal{D}$ and $\operatorname{sdepth}(M)$ is called the Stanley depth of $M$. For an introduction to Stanley depth, we refer the reader to $[7,10,23]$. Stanley conjectured in [26] that $\operatorname{sdepth}(M) \geq \operatorname{depth}(M)$ for any $\mathbb{Z}^{n}$-graded $S$-module $M$. This conjecture was disproved by Duval et al. [6]. However, there still looks to be a deep and interesting relationship between depth and Stanley depth, which is yet to be exactly understood. Also it is interesting to find new classes of modules which satisfy Stanley's inequality because in this case we have a lower bound for the Stanley depth.

\footnotetext{
*Corresponding Author.

Email addresses: 786zahidwarraich@gmail.com (Z. Iqbal), ishaq_maths@yahoo.com (M. Ishaq), ahsanbanyamin@gmail.com (M.A. Binyamin)

Received: 25.10.2019; Accepted: 02.05.2020
} 
Let $I \subset J \subset S$ be monomial ideals, Herzog et al. [11] showed that the invariant Stanley depth of $J / I$ is combinatorial in nature. The strange thing about Stanley depth is that it shares some properties and bounds with homological invariant depth see $([11,15,22,24])$. Until now mathematicians are not too much familiar with Stanley depth as it is hard to compute, for computation and some known results we refer the readers to $([1,12,16,17,19])$. Let $P_{n}$ and $C_{n}$ represent path and cycle respectively on $n$ vertices and $\nabla$ represents the strong product of two graphs. The aim of this paper is to study depth and Stanley depth of the edge ideals and quotient ring of the edge ideals associated with classes of graphs $\mathcal{H}:=\left\{P_{n} \otimes P_{m}: n, m \geq 1\right\}$ and $\mathcal{K}:=\left\{C_{n} \otimes P_{m}: n \geq 3, m \geq 1\right\}$. In Section 3 we compute depth and Stanley depth of quotient ring of edge ideals associated with some subclasses of $\mathcal{H}$ and $\mathcal{K}$. For the monomial ideal $I \subset S$ it is clear that $\operatorname{depth}(I)=\operatorname{depth}(S / I)+1$, this means that once you know about $\operatorname{depth}(S / I)$ then you also know about $\operatorname{depth}(I)$ and vice versa, whereas for Stanley depth this is not the case. So far all examples show that $\operatorname{sdepth}(I) \geq \operatorname{sdepth}(S / I)$, as Herzog conjectured:

Conjecture 1 ([10, Conjecture 64]). Let $I \subset S$ be a monomial ideal then $\operatorname{sdepth}(I) \geq$ $\operatorname{sdepth}(S / I)$.

In Section 4 of this paper, we confirm the above conjecture for the edge ideals associated with some subclasses of $\mathcal{H}$ and $\mathcal{K}$. For recent works on the above conjecture, we refer the reader to $[13,14,18]$. In Section 5, we give sharp upper bounds for depth and Stanley depth of quotient ring of the edge ideals associated to $\mathcal{H}$ and $\mathcal{K}$. In the same section, we also propose some open questions. We gratefully acknowledge the use of the computer algebra system CoCoA ([5]) for our experiments.

\section{Definitions and notations}

In this section, we review some standard terminologies and notations from graph theory and algebra. For more details, one may consult [9,28]. Let $G:=(V(G), E(G))$ be a graph with vertex set $V(G):=\left\{x_{1}, x_{2}, \ldots, x_{n}\right\}$ and edge set $E(G)$. The edge ideal $I(G)$ associated with $G$ is a squarefree monomial ideal of $S$, that is $I(G)=\left(x_{i} x_{j}:\left\{x_{i}, x_{j}\right\} \in\right.$ $E(G))$. A graph $G$ on $n \geq 2$ vertices is called a path on $n$ vertices if $E(G)=\left\{\left\{x_{i}, x_{i+1}\right\}\right.$ : $i=1,2 \ldots, n-1\}$. We denote a path on $n$ vertices by $P_{n}$. A graph $G$ on $n \geq 3$ vertices is called a cycle if $E(G)=\left\{\left\{x_{i}, x_{i+1}\right\}: i=1,2, \ldots, n-1\right\} \cup\left\{\left\{x_{1}, x_{n}\right\}\right\}$. A cycle on $n$ vertices is denoted by $C_{n}$. For vertices $x_{i}$ and $x_{j}$ of a graph $G$, the length of a shortest path from $x_{i}$ to $x_{j}$ is called the distance between $x_{i}$ and $x_{j}$ denoted by $\mathrm{d}_{G}\left(x_{i}, x_{j}\right)$. If no such path exists between $x_{i}$ and $x_{j}$, then $d_{G}\left(x_{i}, x_{j}\right)=\infty$. The diameter of a connected graph $G$ is $\operatorname{diam}(G):=\max \left\{\mathrm{d}_{G}\left(x_{i}, x_{j}\right): x_{i}, x_{j} \in V(G)\right\}$. For a monomial $u, \operatorname{supp}(u):=\left\{x_{i}: x_{i} \mid u\right\}$.

Definition 2.1 ([9]). The strong product $G_{1} \otimes G_{2}$ of graphs $G_{1}$ and $G_{2}$ is a graph, with $V\left(G_{1} \otimes G_{2}\right)=V\left(G_{1}\right) \times V\left(G_{2}\right)$ (the Cartesian product of sets), and for $\left(v_{1}, u_{1}\right),\left(v_{2}, u_{2}\right) \in$ $V\left(G_{1} \otimes G_{2}\right),\left\{\left(v_{1}, u_{1}\right),\left(v_{2}, u_{2}\right)\right\} \in E\left(G_{1} \otimes G_{2}\right)$, whenever

- $\left\{v_{1}, v_{2}\right\} \in E\left(G_{1}\right)$ and $u_{1}=u_{2}$ or

- $v_{1}=v_{2}$ and $\left\{u_{1}, u_{2}\right\} \in E\left(G_{2}\right)$ or

- $\left\{v_{1}, v_{2}\right\} \in E\left(G_{1}\right)$ and $\left\{u_{1}, u_{2}\right\} \in E\left(G_{2}\right)$.

Let $P_{1}$ denote the null graph on one vertex that is $V\left(P_{1}\right):=\left\{x_{1}\right\}$ and $E\left(P_{1}\right):=\emptyset$. Let $\mathcal{P}_{n, m}:=P_{n} \otimes P_{m} \cong P_{m} \otimes P_{n}$, if $n=m=1$, then $\mathcal{P}_{1,1} \cong P_{1}$, this trivial case is excluded. For $n \geq 3$ and $m \geq 1$, let $\mathcal{C}_{n, m}:=C_{n} \otimes P_{m} \cong P_{m} \otimes C_{n}$.

Remark 2.2. $\left|V\left(\mathcal{P}_{n, m}\right)\right|=n m,\left|E\left(\mathcal{P}_{n, m}\right)\right|=4(n-1)(m-1)+(n-1)+(m-1),\left|V\left(\mathfrak{C}_{n, m}\right)\right|=$ $n m$ and $\left|E\left(\mathcal{C}_{n, m}\right)\right|=\left|E\left(\mathcal{P}_{n, m}\right)\right|+3(m-1)+1$.

Since both graphs $\mathcal{P}_{n, m}$ and $\mathcal{C}_{n, m}$ are on $n m$ vertices, for the sake of convenience, we label the vertices of $\mathcal{P}_{n, m}$ and $\mathcal{C}_{n, m}$ by using $m$ sets of variables $\left\{x_{1 j}, x_{2 j}, \ldots, x_{n j}\right\}$ where 
$1 \leq j \leq m$. We set $S_{n, m}:=K\left[\cup_{j=1}^{m}\left\{x_{1 j}, x_{2 j}, \ldots, x_{n j}\right\}\right]$. For examples of $\mathcal{P}_{n, m}$ and $\mathcal{C}_{n, m}$ see Fig 1.
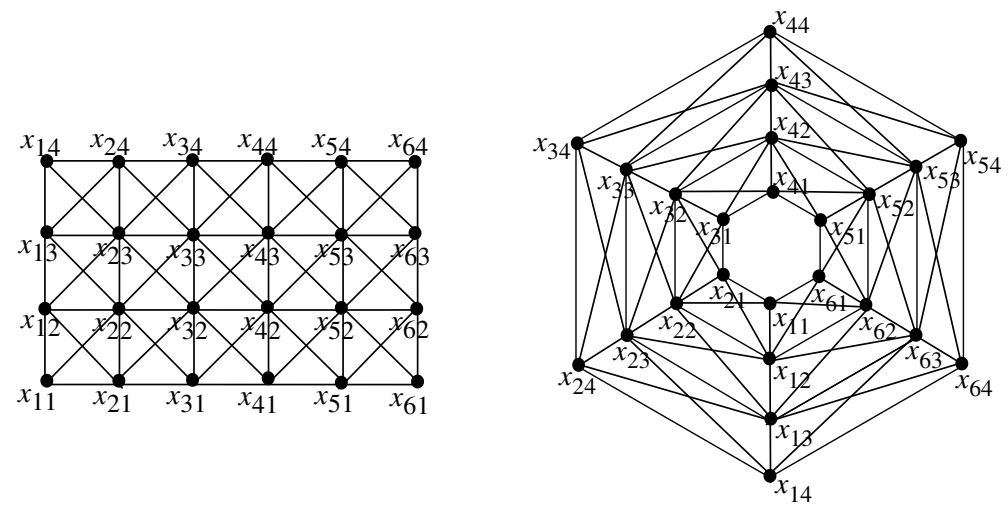

Figure 1. From left to right; $\mathcal{P}_{6,4}$ and $\mathfrak{C}_{6,4}$.

Remark 2.3. Let $\mathcal{G}(I)$ denote the unique minimal set of monomial generators of the monomial ideal $I$.

(1) For positive integers $m, n$ such that $m$ and $n$ are not equal to 1 simultaneously, the minimal set of monomial generators of the edge ideal of $\mathcal{P}_{n, m}$ is given as:

$$
\begin{array}{r}
\mathcal{G}\left(I\left(\mathcal{P}_{n, m}\right)\right)=\cup_{i=1}^{n-1}\left\{\cup_{j=1}^{m-1}\left\{x_{i j} x_{i(j+1)}, x_{i j} x_{(i+1)(j+1)}, x_{i j} x_{(i+1) j}, x_{(i+1) j} x_{i(j+1)}, x_{n j} x_{n(j+1)}\right\},\right. \\
\left.x_{i m} x_{(i+1) m}\right\} .
\end{array}
$$

(2) For $n \geq 3, m \geq 1$, the minimal set of monomial generators for $I\left(\mathcal{C}_{n, m}\right)$ is:

$\mathcal{G}\left(I\left(\mathcal{C}_{n, m}\right)\right)=\mathcal{G}\left(I\left(\mathcal{P}_{n, m}\right)\right) \cup\left\{\cup_{j=1}^{m-1}\left\{x_{1 j} x_{n(j+1)}, x_{1 j} x_{n j}, x_{1(j+1)} x_{n j}\right\}, x_{1 m} x_{n m}\right\}$.

(3) $\mathcal{P}_{n, 1} \cong P_{n}$ and $\mathcal{C}_{n, 1} \cong C_{n}$.

(4) For $n, m \geq 1, \mathcal{P}_{n, m} \cong \mathcal{P}_{m, n}$, so without loss of generality the strong product of two paths can be represented as $\mathcal{P}_{n, m}$ with $m \leq n$. Thus in some proofs by induction on $n$, whenever we are reduced to the case where we have $\mathcal{P}_{n^{\prime}, m}$ with $n^{\prime}<m$, after a suitable relabeling of vertices we have $\mathcal{P}_{n^{\prime}, m} \cong \mathcal{P}_{m, n^{\prime}}$. Therefore, we can simply replace $I\left(\mathcal{P}_{n^{\prime}, m}\right)$ by $I\left(\mathcal{P}_{m, n^{\prime}}\right)$ and $S_{n^{\prime}, m} / I\left(\mathcal{P}_{n^{\prime}, m}\right)$ by $S_{m, n^{\prime}} / I\left(\mathcal{P}_{m, n^{\prime}}\right)$.

The method of Herzog et al. [11] for determining the Stanley depth of modules of the type $M=J / I$ (where $I \subset J \subset S$ are monomial ideals) using posets can be summarized in the following way. We define a natural partial order on $\mathbb{N}^{n}$ as follows: $a \leq b$ if and only if $a(l) \leq b(l)$ for $l=1, \ldots, n$. Note that $x^{a} \mid x^{b}$ if and only if $a \leq b$. Here for $c \in \mathbb{N}^{n}, x^{c}$ denote the monomial $x_{1}^{c(1)} x_{2}^{c(2)} \cdots x_{n}^{c(n)}$. Let $J=\left(x^{a_{1}}, x^{a_{2}}, \ldots, x^{a_{r}}\right)$ and $I=\left(x^{b_{1}}, x^{b_{2}}, \ldots, x^{b_{t}}\right)$ where $a_{i}, b_{j} \in \mathbb{N}^{n}$. Let $h \in \mathbb{N}^{n}$ such that $\left.h(l)=\max \left\{a_{i}(l), b_{j}(l)\right): 1 \leq i \leq r, 1 \leq j \leq t\right\}$ (the component-wise maximum of the $a_{i}$ and $b_{j}$ ). Then the characteristic poset of $J / I$ with respect to $h$, denoted $P_{J / I}^{h}$, is the induced subposet of $\mathbb{N}^{n}$ with ground set

$$
\left\{c \in \mathbb{N}^{n} \mid c \leq h \text {, there is } i \text { such that } c \geq a_{i} \text {, and for all } j, c \nsupseteq b_{j}\right\} \text {. }
$$

Let $x, y \in P_{J / I}^{h}, \alpha:=[x, y]=\left\{z \in P_{J / I}^{h}: x \leq z \leq y\right\}$ be a subset of $P_{J / I}^{h}$ called interval and $\mathbf{P}$ be a partition of $P_{J / I}^{h}$ into intervals. Let $Z_{\alpha}:=\{l: y(l)=h(l)\}$, define the Stanley depth of a partition $\mathbf{P}$ to be $\operatorname{sdepth}(\mathbf{P}):=\min _{\alpha \in \mathbf{P}}\left|Z_{\alpha}\right|$ and the Stanley depth of the poset $P_{J / I}^{h}$ to be $\operatorname{sdepth}\left(P_{J / I}^{h}\right):=\max _{\mathbf{P}} \operatorname{sdepth}(\mathbf{P})$, where the maximum is taken over all partitions $\mathbf{P}$ of $P_{J / I}^{h}$. Herzog et al. showed in [11] that $\operatorname{sdepth}(J / I)=\operatorname{sdepth}\left(P_{J / I}^{h}\right)$. By considering all partitions of the characteristic poset, this correspondence provides an algorithm (albeit inefficient) to find the Stanley depth of $J / I$. Now we recall some known results that are heavily used in this paper. 
Lemma 2.4. (Depth Lemma) If $0 \rightarrow U \rightarrow M \rightarrow N \rightarrow 0$ is a short exact sequence of modules over a local ring $S$, or a Noetherian graded ring with local $S_{0}$, then

(1) $\operatorname{depth}(M) \geq \min \{\operatorname{depth}(N), \operatorname{depth}(U)\}$.

(2) $\operatorname{depth}(U) \geq \min \{\operatorname{depth}(M), \operatorname{depth}(N)+1\}$.

(3) $\operatorname{depth}(N) \geq \min \{\operatorname{depth}(U)-1, \operatorname{depth}(M)\}$.

Lemma 2.5 ([24, Lemma 2.2]). Let $0 \rightarrow U \rightarrow V \rightarrow W \rightarrow 0$ be a short exact sequence of $\mathbb{Z}^{n}$-graded $S$-modules. Then $\operatorname{sdepth}(V) \geq \min \{\operatorname{sdepth}(U), \operatorname{sdepth}(W)\}$.

Remark 2.6. Let $I \subset S$ be a monomial ideal. Then for $1 \leq i \leq n$ with $x_{i} \notin I$, the short exact sequence

$$
0 \longrightarrow S /\left(I: x_{i}\right) \stackrel{\cdot x_{i}}{\longrightarrow} S / I \longrightarrow S /\left(I, x_{i}\right) \longrightarrow 0,
$$

implies that

$$
\begin{gathered}
\operatorname{depth}(S / I) \geq \min \left\{\operatorname{depth}\left(S /\left(I: x_{i}\right)\right), \operatorname{depth}\left(S /\left(I, x_{i}\right)\right)\right\}, \\
\operatorname{sdepth}(S / I) \geq \min \left\{\operatorname{sdepth}\left(S /\left(I: x_{i}\right)\right), \operatorname{sdepth}\left(S /\left(I, x_{i}\right)\right)\right\} .
\end{gathered}
$$

This will be used frequently throughout the paper.

Lemma 2.7 ([11, Lemma 3.6]). Let $I \subset J$ be monomial ideals of $S$ and $\bar{S}=S\left[x_{n+1}\right]$ be a polynomial ring in $n+1$ variables. Then

$$
\operatorname{depth}(J \bar{S} / I \bar{S})=\operatorname{depth}(J S / I S)+1 \quad \text { and } \quad \operatorname{sdepth}(J \bar{S} / I \bar{S})=\operatorname{sdepth}(J S / I S)+1 .
$$

Corollary 2.8 ([24, Corollary 1.3]). Let $J \subset S$ be a monomial ideal. Then $\operatorname{depth}(S / J) \leq$ $\operatorname{depth}(S /(J: v))$ for all monomials $v \notin J$.

Proposition 2.9 ([2, Proposition 2.7]). Let $J \subset S$ be a monomial ideal. Then for all monomials $v \notin J \operatorname{sdepth}(S / J) \leq \operatorname{sdepth}(S /(J: v))$.

Let $q \in \mathbb{Q}$, then $\lceil q\rceil$ denote the smallest integer greater than or equal to $q$, and $\lfloor q\rfloor$ denote the greatest integer less than or equal to $q$.

Theorem 2.10 ([21, Theorem 2.3]). Let $I \subset S$ be a monomial ideal of $S$ and $m$ be the number of minimal monomial generators of $I$, then $\operatorname{sdepth}(I) \geq \max \left\{1, n-\left\lfloor\frac{m}{2}\right\rfloor\right\}$.

Corollary 2.11 ([8, Corollary 3.2]). Let $G$ be a connected graph of diameter $d \geq 1$ and let $I=I(G)$. Then $\operatorname{depth}(S / I) \geq\left\lceil\frac{d+1}{3}\right\rceil$.

Theorem 2.12 ([8, Theorem 4.18]). Let $G$ be a graph with $p$ connected components, $I=I(G)$, and let $d=d(G)$ be the diameter of $G$. Then, for $1 \leq t \leq 3$ we have

$$
\operatorname{sdepth}\left(S / I^{t}\right) \geq\left\lceil\frac{d-4 t+5}{3}\right\rceil+p-1 .
$$

Corollary 2.13. Let $G$ be a connected graph of diameter $d \geq 1$ and let $I=I(G)$. Then $\operatorname{sdepth}(S / I) \geq\left\lceil\frac{d+1}{3}\right\rceil$.

3. Depth and Stanley depth of cyclic modules associated to $\mathcal{P}_{n, m}$ and $\mathcal{C}_{n, m}$ when $1 \leq m \leq 3$

Let $n \geq 2$ and $1 \leq i \leq n$, for convenience we take $x_{i}:=x_{i 1}, y_{i}:=x_{i 2}$ and $z_{i}:=x_{i 3}$, see Figures 2 and 3 . We set $S_{n, 1}:=K\left[x_{1}, x_{2}, \ldots, x_{n}\right], S_{n, 2}:=K\left[x_{1}, x_{2}, \ldots x_{n}, y_{1}, y_{2}, \ldots, y_{n}\right]$ and $S_{n, 3}:=K\left[x_{1}, x_{2}, \ldots x_{n}, y_{1}, y_{2}, \ldots, y_{n}, z_{1}, z_{2}, \ldots, z_{n}\right]$. Clearly $\mathcal{P}_{n, 1} \cong P_{n}$ and $\mathcal{C}_{n, 1} \cong C_{n}$, the minimal sets of monomial generators of the edge ideals of $\mathcal{P}_{n, 2}, \mathcal{P}_{n, 3}, \mathcal{C}_{n, 2}$ and $\mathcal{C}_{n, 3}$ are given as:

$$
\begin{gathered}
\mathcal{G}\left(I\left(\mathcal{P}_{n, 2}\right)\right)=\cup_{i=1}^{n-1}\left\{x_{i} y_{i}, x_{i} y_{i+1}, x_{i} x_{i+1}, x_{i+1} y_{i}, y_{i} y_{i+1}\right\} \cup\left\{x_{n} y_{n}\right\}, \\
\mathcal{G}\left(I\left(\mathcal{P}_{n, 3}\right)\right)=\cup_{i=1}^{n-1}\left\{x_{i} y_{i}, x_{i} y_{i+1}, x_{i} x_{i+1}, x_{i+1} y_{i}, y_{i} y_{i+1}, y_{i} z_{i}, y_{i} z_{i+1}, y_{i+1} z_{i}, z_{i} z_{i+1}\right\} \cup\left\{x_{n} y_{n}, y_{n} z_{n}\right\},
\end{gathered}
$$

$$
\mathcal{G}\left(I\left(\mathcal{C}_{n, 2}\right)\right)=\mathcal{G}\left(I\left(\mathcal{P}_{n, 2}\right)\right) \cup\left\{x_{1} y_{n}, x_{1} x_{n}, y_{1} x_{n}, y_{1} y_{n}\right\} \text { and }
$$




$$
\mathcal{G}\left(I\left(\mathcal{C}_{n, 3}\right)\right)=\mathcal{G}\left(I\left(\mathcal{P}_{n, 3}\right)\right) \cup\left\{x_{1} y_{n}, x_{1} x_{n}, y_{1} x_{n}, y_{1} y_{n}, y_{1} z_{n}, z_{1} y_{n}, z_{1} z_{n}\right\} .
$$

In this section, we compute depth and Stanley depth of the cyclic modules $S_{n, m} / I\left(\mathcal{P}_{n, m}\right)$ and $S_{n, m} / I\left(\mathrm{C}_{n, m}\right)$, when $m=1,2,3$.
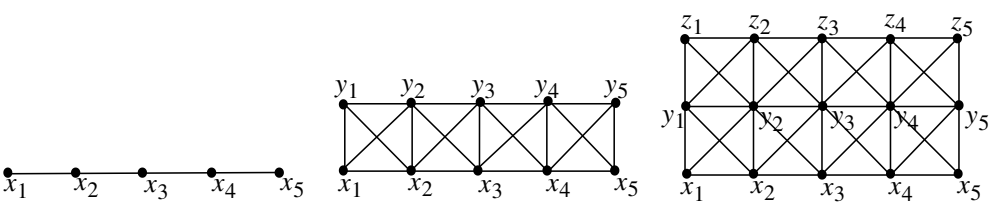

Figure 2. From left to right; $\mathcal{P}_{5,1}, \mathcal{P}_{5,2}$ and $\mathcal{P}_{5,3}$.
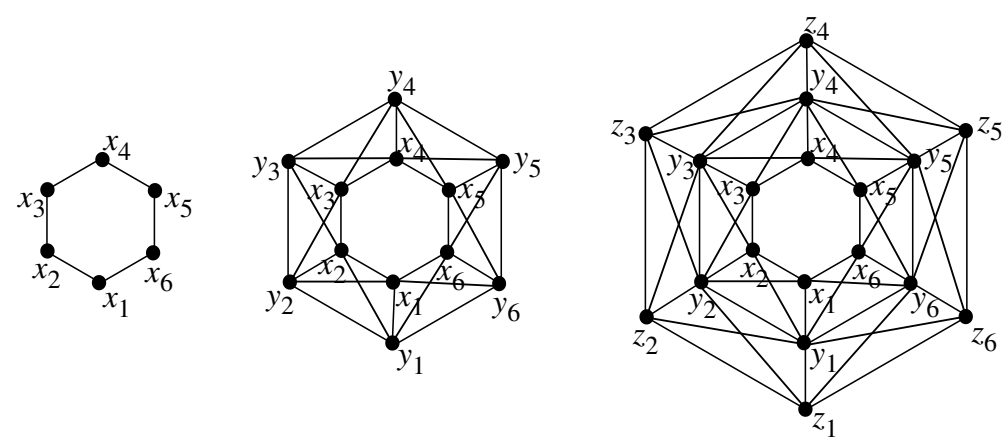

Figure 3. From left to right; $\mathfrak{C}_{6,1}, \mathfrak{C}_{6,2}$ and $\mathfrak{C}_{6,3}$.

Remark 3.1. Note that for $n \geq 2, S_{n, 1} / I\left(\mathcal{P}_{n, 1}\right) \cong S / I\left(P_{n}\right)$, thus by [20, Lemma 2.8] and [27, Lemma 4] $\operatorname{depth}\left(S_{n, 1} / I\left(\mathcal{P}_{n, 1}\right)\right)=\operatorname{sdepth}\left(S_{n, 1} / I\left(\mathcal{P}_{n, 1}\right)\right)=\left\lceil\frac{n}{3}\right\rceil$. Let $n \geq 3$, then $S_{n, 1} / I\left(\mathcal{C}_{n, 1}\right) \cong S / I\left(C_{n}\right)$, and by [4, Propositions 1.3,1.8] $\operatorname{depth}\left(S_{n, 1} / I\left(\mathcal{C}_{n, 1}\right)\right)=\left\lceil\frac{n-1}{3}\right\rceil \leq$ $\operatorname{sdepth}\left(S_{n, 1} / I\left(\mathrm{C}_{n, 1}\right)\right) \leq\left\lceil\frac{n}{3}\right\rceil$.

Lemma 3.2. For $n \geq 1$ and $m=2,3, \operatorname{depth}\left(S_{n, m} / I\left(\mathcal{P}_{n, m}\right)\right)=\operatorname{sdepth}\left(S_{n, m} / I\left(\mathcal{P}_{n, m}\right)\right)=$ $\left\lceil\frac{n}{3}\right\rceil$.

Proof. If $n=1$, then proof follows from Remark 3.1. Let $n \geq 2$. First we prove the result for depth. If $(n, m) \in\{(2,2),(3,2),(3,3)\}$ then the result is trivial. Let $n \geq 4$. $\operatorname{Since} \operatorname{diam}\left(\mathcal{P}_{n, m}\right)=n-1$, thus by Corollary $2.11 \operatorname{depth}\left(S_{n, m} / I\left(\mathcal{P}_{n, m}\right)\right) \geq\left\lceil\frac{n}{3}\right\rceil$. Now we prove that $\operatorname{depth}\left(S_{n, m} / I\left(\mathcal{P}_{n, m}\right)\right) \leq\left\lceil\frac{n}{3}\right\rceil$, we prove this inequality by induction on $n$. Since $y_{n-1} \notin I\left(\mathcal{P}_{n, m}\right)$, then by Corollary 2.8

$$
\operatorname{depth}\left(S_{n, m} / I\left(\mathcal{P}_{n, m}\right)\right) \leq \operatorname{depth}\left(S_{n, m} /\left(I\left(\mathcal{P}_{n, m}\right): y_{n-1}\right)\right) .
$$

As we can see that $S_{n, m} /\left(I\left(\mathcal{P}_{n, m}\right): y_{n-1}\right) \cong S_{n-3, m} / I\left(\mathcal{P}_{n-3, m}\right)\left[y_{n-1}\right]$, therefore by induction and Lemma $2.7 \operatorname{depth}\left(S_{n, m} /\left(I\left(\mathcal{P}_{n, m}\right): y_{n-1}\right)\right)=\left\lceil\frac{n-3}{3}\right\rceil+1=\left\lceil\frac{n}{3}\right\rceil$. This completes the proof for depth.

Now we prove the result for Stanley depth. If $n=m=2$, then $I\left(\mathcal{P}_{2,2}\right)$ is a squarefree Veronese ideal of degree 2. Thus by [3, Theorem 1.1] we have $\operatorname{sdepth}\left(S_{n, 2} / I\left(\mathcal{P}_{n, 2}\right)\right)=1$, as required. If $n=3$ and $m=2$ or 3 , then $\operatorname{diam}\left(\mathcal{P}_{3, m}\right)=2$, thus by Corollary 2.13, we have $\operatorname{sdepth}\left(S_{3, m} / I\left(\mathcal{P}_{3, m}\right)\right) \geq 1$. By Proposition 2.9 we have $\operatorname{sdepth}\left(S_{3, m} / I\left(\mathcal{P}_{3, m}\right)\right) \leq$ $\operatorname{sdepth}\left(S_{3, m} /\left(I\left(\mathcal{P}_{3, m}\right): y_{2}\right)\right)$ it is easy to see that $S_{3, m} /\left(I\left(\mathcal{P}_{3, m}\right): y_{2}\right) \cong K\left[y_{2}\right]$, therefore $\operatorname{sdepth}\left(S_{3, m} / I\left(\mathcal{P}_{3, m}\right)\right) \leq 1$, thus $\operatorname{sdepth}\left(S_{3, m} / I\left(\mathcal{P}_{3, m}\right)\right)=1$. Let $n \geq 4$, using Corollary 2.13 instead of Corollary 2.11 and Proposition 2.9 instead of Corollary 2.8, the proof for depth also works for Stanley depth. 
Theorem 3.3. For $n \geq 3$, $\operatorname{sdepth}\left(S_{n, 2} / I\left(\mathfrak{C}_{n, 2}\right)\right) \geq \operatorname{depth}\left(S_{n, 2} / I\left(\mathcal{C}_{n, 2}\right)\right)=\left\lceil\frac{n-1}{3}\right\rceil$.

Proof. We first prove that $\operatorname{depth}\left(S_{n, 2} / I\left(\mathfrak{C}_{n, 2}\right)\right)=\left\lceil\frac{n-1}{3}\right\rceil$. For $n=3,4$ the result is trivial. For $n \geq 5$ using Remark 2.6 one has

$$
\begin{gathered}
\operatorname{depth}\left(S_{n, 2} / I\left(\mathcal{C}_{n, 2}\right)\right) \geq \min \left\{\operatorname{depth}\left(S_{n, 2} /\left(I\left(\mathcal{C}_{n, 2}\right): x_{n}\right)\right), \operatorname{depth}\left(S_{n, 2} /\left(I\left(\mathcal{C}_{n, 2}\right), x_{n}\right)\right)\right\} . \\
\left(I\left(\mathfrak{C}_{n, 2}\right): x_{n}\right)=\left(\cup_{i=2}^{n-3}\left\{x_{i} y_{i}, x_{i} y_{i+1}, x_{i} x_{i+1}, x_{i+1} y_{i}, y_{i} y_{i+1}\right\}, x_{n-2} y_{n-2}, x_{1}, y_{1}, x_{n-1}, y_{n-1}, y_{n}\right) .
\end{gathered}
$$

After renumbering the variables, we have $S_{n, 2} /\left(I\left(\mathcal{C}_{n, 2}\right): x_{n}\right) \cong S_{n-3,2} / I\left(\mathcal{P}_{n-3,2}\right)\left[x_{n}\right]$. Thus by Lemmas 3.2 and $2.7 \operatorname{depth}\left(S_{n, 2} /\left(I\left(\mathcal{C}_{n, 2}\right): x_{n}\right)\right)=\left\lceil\frac{n-3}{3}\right\rceil+1=\left\lceil\frac{n}{3}\right\rceil$. Let $J$ be a monomial ideal such that;

$$
\begin{aligned}
J=\left(I\left(\mathcal{C}_{n, 2}\right), x_{n}\right)= & \left(\cup_{i=1}^{n-2}\left\{x_{i} y_{i}, x_{i} y_{i+1}, x_{i} x_{i+1}, x_{i+1} y_{i}, y_{i} y_{i+1}\right\}, x_{n-1} y_{n-1}, x_{n}, x_{n-1} y_{n},\right. \\
& \left.y_{n-1} y_{n}, y_{1} y_{n}, x_{1} y_{n}\right)=\left(I\left(\mathcal{P}_{n-1,2}\right), x_{n}, x_{n-1} y_{n}, y_{n-1} y_{n}, y_{1} y_{n}, x_{1} y_{n}\right) .
\end{aligned}
$$

By Remark 2.6 we have $\operatorname{depth}\left(S_{n, 2} / J\right) \geq \min \left\{\operatorname{depth}\left(S_{n, 2} /\left(J: y_{n}\right)\right), \operatorname{depth}\left(S_{n, 2} /\left(J, y_{n}\right)\right)\right\}$. As $\left(J, y_{n}\right)=\left(I\left(\mathcal{P}_{n-1,2}\right), x_{n}, y_{n}\right)$ and $S_{n, 2} /\left(J, y_{n}\right) \cong S_{n-1,2} / I\left(\mathcal{P}_{n-1,2}\right)$. Therefore by Lemma $3.2 \operatorname{depth}\left(S_{n, 2} /\left(J, y_{n}\right)\right)=\left\lceil\frac{n-1}{3}\right\rceil$. Also

$$
\left(J: y_{n}\right)=\left(\cup_{i=2}^{n-3}\left\{x_{i} y_{i}, x_{i} y_{i+1}, x_{i} x_{i+1}, x_{i+1} y_{i}, y_{i} y_{i+1}\right\}, x_{n-2} y_{n-2}, x_{1}, y_{1}, x_{n-1}, y_{n-1}, x_{n}\right) .
$$

After renumbering the variables, we get $S_{n, 2} /\left(J: y_{n}\right) \cong S_{n-3,2} / I\left(\mathcal{P}_{n-3,2}\right)\left[y_{n}\right]$. Therefore by Lemmas 3.2 and $2.7 \operatorname{depth}\left(S_{n, 2} /\left(J: y_{n}\right)\right)=\left\lceil\frac{n-3}{3}\right\rceil+1=\left\lceil\frac{n}{3}\right\rceil$. If $n \equiv 0(\bmod 3)$ or $n \equiv$ $2(\bmod 3)$, then $\operatorname{depth}\left(S_{n, 2} /\left(I\left(\mathcal{C}_{n, 2}\right): x_{n}\right)\right)=\left\lceil\frac{n}{3}\right\rceil=\left\lceil\frac{n-1}{3}\right\rceil \leq \operatorname{depth}\left(S_{n, 2} /\left(I\left(\mathfrak{C}_{n, 2}\right), x_{n}\right)\right)$, thus Depth Lemma implies $\operatorname{depth}\left(S_{n, 2} / I\left(\complement_{n, 2}\right)\right)=\left\lceil\frac{n-1}{3}\right\rceil$, as required. Now for $n \equiv$ $1(\bmod 3)$, assume that $n \geq 7$, then we have the following $S_{n, 2}$-module isomorphism:

$$
\begin{gathered}
\left(I\left(\mathcal{C}_{n, 2}\right): x_{n}\right) / I\left(\mathcal{C}_{n, 2}\right) \cong x_{1} \frac{K\left[x_{3}, \ldots, x_{n-1}, y_{3}, \ldots, y_{n-1}\right]}{\left(\bigcup_{i=3}^{n-2}\left\{x_{i} y_{i}, x_{i} y_{i+1}, x_{i} x_{i+1}, x_{i+1} y_{i}, y_{i} y_{i+1}\right\}, x_{n-1} y_{n-1}\right)}\left[x_{1}\right] \\
\oplus y_{1} \frac{K\left[x_{3}, \ldots, x_{n-1}, y_{3}, \ldots, y_{n-1}\right]}{\left(\bigcup_{i=3}^{n-2}\left\{x_{i} y_{i}, x_{i} y_{i+1}, x_{i} x_{i+1}, x_{i+1} y_{i}, y_{i} y_{i+1}\right\}, x_{n-1} y_{n-1}\right)}\left[y_{1}\right] \\
\oplus y_{n} \frac{K\left[x_{2}, \ldots, x_{n-2}, y_{2}, \ldots, y_{n-2}\right]}{\left(\bigcup_{i=2}^{n-3}\left\{x_{i} y_{i}, x_{i} y_{i+1}, x_{i} x_{i+1}, x_{i+1} y_{i}, y_{i} y_{i+1}\right\}, x_{n-2} y_{n-2}\right)}\left[y_{n}\right] \\
\oplus x_{n-1} \frac{K\left[x_{2}, \ldots, x_{n-3}, y_{2}, \ldots, y_{n-3}\right]}{\left(\bigcup_{i=2}^{n-4}\left\{x_{i} y_{i}, x_{i} y_{i+1}, x_{i} x_{i+1}, x_{i+1} y_{i}, y_{i} y_{i+1}\right\}, x_{n-3} y_{n-3}\right)}\left[x_{n-1}\right] \\
\oplus y_{n-1} \frac{K\left[x_{2}, \ldots, x_{n-3}, y_{2}, \ldots, y_{n-3}\right]}{\left(\bigcup_{i=2}^{n-4}\left\{x_{i} y_{i}, x_{i} y_{i+1}, x_{i} x_{i+1}, x_{i+1} y_{i}, y_{i} y_{i+1}\right\}, x_{n-3} y_{n-3}\right)}\left[y_{n-1}\right] .
\end{gathered}
$$

Indeed, if $u \in\left(I\left(\mathcal{C}_{n, 2}\right): x_{n}\right)$ is a monomial such that $u \notin I\left(\mathfrak{C}_{n, 2}\right)$. Then $u$ is divisible by at most one variable from the set $\left\{x_{1}, y_{1}, y_{n}, x_{n-1}, y_{n-1}\right\}$, if $u$ is divisible by two or more variables from $\left\{x_{1}, y_{1}, y_{n}, x_{n-1}, y_{n-1}\right\}$ then $u \in I\left(\mathcal{C}_{n, 2}\right)$, a contradiction. If $x_{1} \mid u$ then $u=$ $x_{1}^{a} w$ with $a \geq 1$, since $u \notin I\left(\mathfrak{C}_{n, 2}\right)$ it follows that $w \in S^{\prime}:=K\left[x_{3}, \ldots, x_{n-1}, y_{3}, \ldots, y_{n-1}\right]$ and $w \notin J:=\left(\bigcup_{i=3}^{n-2}\left\{x_{i} y_{i}, x_{i} y_{i+1}, x_{i} x_{i+1}, x_{i+1} y_{i}, y_{i} y_{i+1}\right\}, x_{n-1} y_{n-1}\right)$, thus $u \in x_{1}\left(S^{\prime} / J\right)\left[x_{1}\right]$ which is the first summand in the direct sum. Let $S^{\prime \prime}:=S^{\prime}\left[x_{1}\right]$ then $x_{1}\left(S^{\prime} / J\right)\left[x_{1}\right] \cong$ $x_{1}\left(S^{\prime \prime} / J S^{\prime \prime}\right)$, it is easy to see that $x_{1}$ is regular on $S^{\prime \prime} / J S^{\prime \prime}$, therefore we have the $S^{\prime \prime}$ module isomorphism $x_{1}\left(S^{\prime \prime} / J S^{\prime \prime}\right)=\left(S^{\prime \prime} / J S^{\prime \prime}\right)$. After a suitable renumbering of variables we have $\left(S^{\prime \prime} / J S^{\prime \prime}\right) \cong S_{n-3,2} / I\left(\mathcal{P}_{n-3,2}\right)\left[x_{n}\right]$. If $y_{1} \mid u$, then we get the second summand and if $y_{n} \mid u$ then we get the third summand. Proceeding in the same way one can easily show that these two summands are also isomorphic to $S_{n-3,2} / I\left(\mathcal{P}_{n-3,2}\right)\left[x_{n}\right]$. If $x_{n-1} \mid u$ then we get the forth summand and if $y_{n-1} \mid u$ then we get the last summand. Similarly one can show that the last two summands are isomorphic to $S_{n-4,2} / I\left(\mathcal{P}_{n-4,2}\right)\left[x_{n}\right]$. Thus by Lemmas 3.2 and 2.7, we have

$$
\left.\operatorname{depth}\left(I\left(\mathfrak{e}_{n, 2}\right): x_{n}\right) / I\left(\mathfrak{e}_{n, 2}\right)\right)=\min \left\{\left\lceil\frac{n-3}{3}\right\rceil+1,\left\lceil\frac{n-4}{3}\right\rceil+1\right\}=\left\lceil\frac{n-1}{3}\right\rceil .
$$


Now by using Depth Lemma on the following short exact sequence we get the required result.

$$
0 \longrightarrow\left(I\left(\mathcal{C}_{n, 2}\right): x_{n}\right) / I\left(\mathcal{C}_{n, 2}\right) \stackrel{x_{n}}{\longrightarrow} S_{n, 2} / I\left(\mathcal{C}_{n, 2}\right) \longrightarrow S_{n, 2} /\left(I\left(\mathcal{C}_{n, 2}\right): x_{n}\right) \longrightarrow 0 .
$$

Now we prove the result for Stanley depth. If $n=3$, then $I\left(\mathcal{C}_{3,2}\right)$ is a squarefree Veronese ideal of degree 2. Thus by [3, Theorem 1.1] $\operatorname{sdepth}\left(S_{3,2} / I\left(\mathcal{C}_{3,2}\right)\right)=1$, as required. If $n=4$, then by using [11] we have the following Stanley decomposition

$$
\begin{aligned}
S_{4,2} / I\left(\mathfrak{C}_{4,2}\right)=K\left[x_{1}, x_{3}\right] \oplus y_{1} K\left[x_{3}, y_{1}\right] \oplus x_{2} K\left[x_{2}, x_{4}\right] \oplus y_{2} K\left[y_{2}, y_{4}\right] \oplus \\
y_{3} K\left[x_{1}, y_{3}\right] \oplus x_{4} K\left[x_{4}, y_{2}\right] \oplus y_{4} K\left[x_{2}, y_{4}\right] \oplus y_{1} y_{3} K\left[y_{1}, y_{3}\right] .
\end{aligned}
$$

Thus $\operatorname{sdepth}\left(S_{4,2} / I\left(\mathrm{C}_{4,2}\right)\right) \geq 2$. For upper bound by Proposition 2.9 we have

$$
\operatorname{sdepth}\left(S_{4,2} / I\left(\mathcal{C}_{4,2}\right)\right) \leq \operatorname{sdepth}\left(S_{4,2} /\left(I\left(\mathcal{C}_{4,2}\right): x_{1} x_{3}\right)\right),
$$

since $S_{4,2} /\left(I\left(\mathcal{C}_{4,2}\right): x_{1} x_{3}\right) \cong K\left[x_{1}, x_{3}\right]$, therefore $\operatorname{sdepth}\left(S_{4,2} / I\left(\mathcal{C}_{4,2}\right)\right) \leq 2$, thus we get $\operatorname{sdepth}\left(S_{4,2} / I\left(\mathcal{C}_{4,2}\right)\right)=2$. Let $n \geq 5$, using Remark 2.6 we have

$$
\operatorname{sdepth}\left(S_{n, 2} / I\left(\mathfrak{C}_{n, 2}\right)\right) \geq
$$

$\min \left\{\operatorname{sdepth}\left(S_{n, 2} /\left(I\left(\mathcal{C}_{n, 2}\right): x_{n}\right)\right), \operatorname{sdepth}\left(S_{n, 2} /\left(J: y_{n}\right)\right), \operatorname{sdepth}\left(S_{n, 2} /\left(J, y_{n}\right)\right)\right\} \geq\left\lceil\frac{n-1}{3}\right\rceil$

Corollary 3.4. For $n \geq 3$, $\left\lceil\frac{n-1}{3}\right\rceil \leq \operatorname{sdepth}\left(S_{n, 2} / I\left(\mathcal{C}_{n, 2}\right)\right) \leq\left\lceil\frac{n}{3}\right\rceil$.

Proof. Since $I\left(\mathcal{C}_{3,2}\right)$ is a squarefree Veronese ideal, by using [3, Theorem 1.1], it follows that $\operatorname{sdepth}\left(S_{3,2} / I\left(\mathfrak{C}_{3,2}\right)\right)=1$. For $n \geq 4$, by Proposition $2.9 \operatorname{sdepth}\left(S_{n, 2} / I\left(\mathfrak{C}_{n, 2}\right)\right) \leq$ $\operatorname{sdepth}\left(S_{n, 2} /\left(I\left(\mathcal{C}_{n, 2}\right): x_{n}\right)\right)$. Since $S_{n, 2} /\left(I\left(\mathcal{C}_{n, 2}\right): x_{n}\right) \cong S_{n-3,2} / I\left(\mathcal{P}_{n-3,2}\right)\left[x_{n}\right]$, using Lemmas 3.2 and 2.7, we have $\operatorname{sdepth}\left(S_{n, 2} /\left(I\left(\mathrm{C}_{n, 2}\right): x_{n}\right)\right)=\left\lceil\frac{n-3}{3}\right\rceil+1=\left\lceil\frac{n}{3}\right\rceil$.

For $n \geq 2$ we define a supergraph of $\mathcal{P}_{n, 3}$ denoted by $\mathcal{P}_{n, 3}^{\star}$ with the set of vertices $V\left(\mathcal{P}_{n, 3}^{\star}\right):=V\left(\mathcal{P}_{n, 3}\right) \cup\left\{z_{n+1}\right\}$ and edge set $E\left(\mathcal{P}_{n, 3}^{\star}\right):=E\left(\mathcal{P}_{n, 3}\right) \cup\left\{z_{n} z_{n+1}, y_{n} z_{n+1}\right\}$. Also we define a supergraph of $\mathcal{P}_{n, 3}^{\star}$ denoted by $\mathcal{P}_{n, 3}^{\star \star}$ with the set of vertices $V\left(\mathcal{P}_{n, 3}^{\star \star}\right):=V\left(\mathcal{P}_{n, 3}^{\star}\right) \cup$ $\left\{z_{n+2}\right\}$ and edge set $E\left(\mathcal{P}_{n, 3}^{\star \star}\right):=E\left(\mathcal{P}_{n, 3}^{\star}\right) \cup\left\{z_{1} z_{n+2}, y_{1} z_{n+2}\right\}$. For examples of $\mathcal{P}_{n, 3}^{\star}$ and $\mathcal{P}_{n, 3}^{\star \star}$ see Fig. 4. Let $S_{n, 3}^{\star}:=S_{n, 3}\left[z_{n+1}\right]$ and $S_{n, 3}^{\star \star}:=S_{n, 3}\left[z_{n+1}, z_{n+2}\right]$ then we have the following lemma:
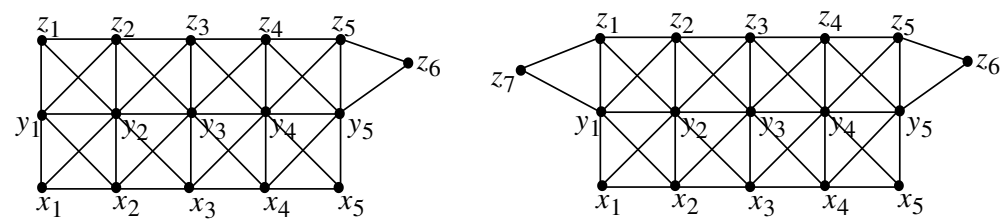

Figure 4. From left to right; $\mathcal{P}_{5,3}^{\star}$ and $\mathcal{P}_{5,3}^{\star \star}$.

Lemma 3.5. For $n \geq 2$,

(a) $\operatorname{depth}\left(S_{n, 3}^{\star} / I\left(\mathcal{P}_{n, 3}^{\star}\right)\right)=\operatorname{sdepth}\left(S_{n, 3}^{\star} / I\left(\mathcal{P}_{n, 3}^{\star}\right)\right)=\left\lceil\frac{n+1}{3}\right\rceil$.

(b) $\operatorname{depth}\left(S_{n, 3}^{\star \star} / I\left(\mathcal{P}_{n, 3}^{\star \star}\right)\right)=\operatorname{sdepth}\left(S_{n, 3}^{\star \star} / I\left(\mathcal{P}_{n, 3}^{\star \star}\right)\right)=\left\lceil\frac{n+2}{3}\right\rceil$.

Proof. (a). First we prove the result for depth. Since $\operatorname{diam}\left(\mathcal{P}_{n, 3}^{\star}\right)=n$, then by Corollary 2.11 we have $\operatorname{depth}\left(S_{n, 3}^{\star} / I\left(\mathcal{P}_{n, 3}^{\star}\right)\right) \geq\left\lceil\frac{n+1}{3}\right\rceil$. Now we prove the reverse inequality, if $n=2$ then the result is trivial. For $n \geq 3$, as $y_{n} \notin I\left(\mathcal{P}_{n, 3}^{\star}\right)$ so by Corollary $2.8 \operatorname{depth}\left(S_{n, 3}^{\star} / I\left(\mathcal{P}_{n, 3}^{\star}\right)\right) \leq \operatorname{depth}\left(S_{n, 3}^{\star} /\left(I\left(\mathcal{P}_{n, 3}^{\star}\right): y_{n}\right)\right)$. We have $S_{n, 3}^{\star} /\left(I\left(\mathcal{P}_{n, 3}^{\star}\right): y_{n}\right) \cong$ $\left(S_{n-2,3} / I\left(\mathcal{P}_{n-2,3}\right)\right)\left[y_{n}\right]$. By Lemmas 3.2 and $2.7 \operatorname{depth}\left(S_{n, 3}^{\star} /\left(I\left(\mathcal{P}_{n, 3}^{\star}\right): y_{n}\right)\right)=\left\lceil\frac{n-2}{3}\right\rceil+1=$ $\left\lceil\frac{n+1}{3}\right\rceil$. Thus $\operatorname{depth}\left(S_{n, 3}^{\star} / I\left(\mathcal{P}_{n, 3}^{\star}\right)\right) \leq\left\lceil\frac{n+1}{3}\right\rceil$. Proof for Stanley depth is similar by using 
Proposition 2.9 and Corollary 2.13.

(b). Clearly $\operatorname{diam}\left(\mathcal{P}_{n, 3}^{\star \star}\right)=n+1$, by Corollary 2.11 we have $\operatorname{depth}\left(S_{n, 3}^{\star \star} / I\left(\mathcal{P}_{n, 3}^{\star \star}\right)\right) \geq\left\lceil\frac{n+2}{3}\right\rceil$. Now we prove the reverse inequality, it is true when $n=2,3$. For $n \geq 4$, as $y_{n} \notin I\left(\mathcal{P}_{n, 3}^{\star \star}\right)$ so by Corollary $2.8 \operatorname{depth}\left(S_{n, 3}^{\star \star} / I\left(\mathcal{P}_{n, 3}^{\star \star}\right)\right) \leq \operatorname{depth}\left(S_{n, 3}^{\star \star} /\left(I\left(\mathcal{P}_{n, 3}^{\star \star}\right): y_{n}\right)\right)$. Since $S_{n, 3}^{\star \star} /\left(I\left(\mathcal{P}_{n, 3}^{\star \star}\right): y_{n}\right) \cong\left(S_{n-2,3}^{\star} / I\left(\mathcal{P}_{n-2,3}^{\star}\right)\right)\left[y_{n}\right]$. By (a) and Lemma 2.7 we obtain $\operatorname{depth}\left(S_{n, 3}^{\star} / I\left(\mathcal{P}_{n, 3}^{\star}\right): y_{n}\right)=\left\lceil\frac{n-2+1}{3}\right\rceil+1=\left\lceil\frac{n+2}{3}\right\rceil$. Thus $\operatorname{depth}\left(S_{n, 3}^{\star \star} / I\left(\mathcal{P}_{n, 3}^{\star \star}\right)\right) \leq\left\lceil\frac{n+2}{3}\right\rceil$. Similarly one can prove the result for Stanley depth by using Proposition 2.9 and Corollary 2.13 .

Theorem 3.6. For $n \geq 3$, and $n \equiv 0,2(\bmod 3), \operatorname{sdepth}\left(S_{n, 3} / I\left(\mathfrak{C}_{n, 3}\right)\right)=\left\lceil\frac{n-1}{3}\right\rceil=$ $\operatorname{depth}\left(S_{n, 3} / I\left(\mathfrak{C}_{n, 3}\right)\right)$, and otherwise, $\left\lceil\frac{n-1}{3}\right\rceil \leq \operatorname{depth}\left(S_{n, 3} / I\left(\mathfrak{C}_{n, 3}\right)\right), \operatorname{sdepth}\left(S_{n, 3} / I\left(\mathfrak{C}_{n, 3}\right)\right) \leq$ $\left\lceil\frac{n}{3}\right\rceil$.

Proof. We first prove the result for depth. For $n=3,4$ the result is clear. Let $n \geq 5$,

$$
\begin{aligned}
& A:=\left(I\left(\mathcal{C}_{n, 3}\right): x_{n}\right)=\left(\cup_{i=2}^{n-3}\left\{x_{i} y_{i}, x_{i} y_{i+1}, x_{i} x_{i+1}, x_{i+1} y_{i}, y_{i} y_{i+1}, y_{i} z_{i}, y_{i} z_{i+1}, y_{i+1} z_{i}, z_{i} z_{i+1}\right\},\right. \\
& \left.x_{n-2} y_{n-2}, y_{n-2} z_{n-2}, x_{1}, y_{1}, x_{n-1}, y_{n-1}, y_{n}, z_{n} z_{n-1}, z_{n-1} z_{n-2}, y_{n-2} z_{n-1}, z_{n} z_{1}, z_{1} z_{2}, y_{2} z_{1}\right),
\end{aligned}
$$

and

$$
\begin{aligned}
& \bar{A}:=\left(I\left(\mathcal{C}_{n, 3}\right), x_{n}\right)=\left(\cup_{i=1}^{n-2}\left\{x_{i} y_{i}, x_{i} y_{i+1}, x_{i} x_{i+1}, x_{i+1} y_{i}, y_{i} y_{i+1}, y_{i} z_{i}, y_{i} z_{i+1}, y_{i+1} z_{i}, z_{i} z_{i+1}\right\}, x_{n},\right. \\
& \left.x_{n-1} y_{n-1}, y_{n-1} z_{n-1}, x_{n-1} y_{n}, y_{n-1} y_{n}, y_{n} z_{n-1}, y_{n-1} z_{n}, z_{n-1} z_{n}, y_{n} z_{n}, y_{1} y_{n}, x_{1} y_{n}, y_{1} z_{n}, y_{n} z_{1}, z_{1} z_{n}\right) \\
& \quad=\left(I\left(\mathcal{P}_{n-1,3}\right), x_{n}, x_{n-1} y_{n}, y_{n-1} y_{n}, y_{n} z_{n-1}, y_{n-1} z_{n}, z_{n-1} z_{n}, y_{n} z_{n}, y_{1} y_{n}, x_{1} y_{n}, y_{1} z_{n}, y_{n} z_{1}, z_{1} z_{n}\right),
\end{aligned}
$$

then by Remark 2.6 we have

$$
\operatorname{depth}\left(S_{n, 3} / I\left(\mathfrak{C}_{n, 3}\right)\right) \geq \min \left\{\operatorname{depth}\left(S_{n, 3} / A\right), \operatorname{depth}\left(S_{n, 3} / \bar{A}\right)\right\} .
$$

Since $\left(A, z_{n}\right)=\left(\cup_{i=2}^{n-3}\left\{x_{i} y_{i}, x_{i} y_{i+1}, x_{i} x_{i+1}, x_{i+1} y_{i}, y_{i} y_{i+1}, y_{i} z_{i}, y_{i} z_{i+1}, y_{i+1} z_{i}, z_{i} z_{i+1}\right\}\right.$,

$$
\left.x_{n-2} y_{n-2}, y_{n-2} z_{n-2}, x_{1}, y_{1}, x_{n-1}, y_{n-1}, y_{n}, z_{n}, z_{n-1} z_{n-2}, y_{n-2} z_{n-1}, z_{1} z_{2}, y_{2} z_{1}\right) \text {, }
$$

after renumbering the variables we have $S_{n, 3} /\left(A, z_{n}\right) \cong\left(S_{n-3,3}^{\star \star} / I\left(\mathcal{P}_{n-3,3}^{\star \star}\right)\right)\left[x_{n}\right]$. Thus by Lemmas 3.5 and $2.7 \operatorname{depth}\left(S_{n, 3} /\left(A, z_{n}\right)\right)=\left\lceil\frac{n-3+2}{3}\right\rceil+1=\left\lceil\frac{n-1}{3}\right\rceil+1$. Also

$$
\begin{array}{r}
\left(A: z_{n}\right)=\left(\cup_{i=2}^{n-3}\left\{x_{i} y_{i}, x_{i} y_{i+1}, x_{i} x_{i+1}, x_{i+1} y_{i}, y_{i} y_{i+1}, y_{i} z_{i}, y_{i} z_{i+1}, y_{i+1} z_{i}, z_{i} z_{i+1}\right\}, x_{n-2} y_{n-2},\right. \\
\left.y_{n-2} z_{n-2}, x_{1}, y_{1}, x_{n-1}, y_{n-1}, y_{n}, z_{n-1}, z_{1}\right),
\end{array}
$$

after renumbering the variables we get $S_{n, 3} /\left(A: z_{n}\right) \cong\left(S_{n-3,3} / I\left(\mathcal{P}_{n-3,3}\right)\right)\left[x_{n}, z_{n}\right]$. Thus by Lemmas 3.2 and $2.7 \operatorname{depth}\left(S_{n, 3} /\left(A: z_{n}\right)\right)=\left\lceil\frac{n-3}{3}\right\rceil+2=\left\lceil\frac{n}{3}\right\rceil+1$. Using Remark 2.6

$$
\begin{aligned}
& \operatorname{depth}\left(S_{n, 3} /(A)\right) \geq \\
& \quad \min \left\{\operatorname{depth}\left(S_{n, 3} /\left(A: z_{n}\right)\right), \operatorname{depth}\left(S_{n, 3} /\left(A, z_{n}\right)\right)\right\}=\min \left\{\left\lceil\frac{n}{3}\right\rceil+1,\left\lceil\frac{n-1}{3}\right\rceil+1\right\} .
\end{aligned}
$$

As $\left(\bar{A}: y_{n}\right)=\left(\cup_{i=2}^{n-3}\left\{x_{i} y_{i}, x_{i} y_{i+1}, x_{i} x_{i+1}, x_{i+1} y_{i}, y_{i} y_{i+1}, y_{i} z_{i}, y_{i} z_{i+1}, y_{i+1} z_{i}, z_{i} z_{i+1}\right\}\right.$,

$$
\left.x_{n-2} y_{n-2}, y_{n-2} z_{n-2}, x_{n}, x_{1}, y_{1}, z_{1}, x_{n-1}, y_{n-1}, z_{n-1}, z_{n}\right),
$$

after renumbering the variables we get $S_{n, 3} /\left(\bar{A}: y_{n}\right) \cong S_{n-3,3} / I\left(\mathcal{P}_{n-3,3}\right)\left[y_{n}\right]$. Therefore by Lemmas 3.2 and $2.7 \operatorname{depth}\left(S_{n, 3} /\left(\bar{A}: y_{n}\right)\right)=\left\lceil\frac{n-3}{3}\right\rceil+1=\left\lceil\frac{n}{3}\right\rceil$. Now let

$$
\begin{aligned}
\widehat{A}:=\left(\bar{A}, y_{n}\right)= & \left(I\left(\mathcal{P}_{n-1,3}\right), x_{n}, y_{n}, y_{n-1} z_{n}, z_{n-1} z_{n}, y_{1} z_{n}, z_{1} z_{n}\right), \\
\operatorname{depth}\left(S_{n, 3} / \bar{A}\right) & \geq \min \left\{\operatorname{depth}\left(S_{n, 3} /\left(\bar{A}: y_{n}\right)\right), \operatorname{depth}\left(S_{n, 3} / \widehat{A}\right)\right\} \\
& =\min \left\{\left\lceil\frac{n}{3}\right\rceil, \operatorname{depth}\left(S_{n, 3} / \widehat{A}\right)\right\} .
\end{aligned}
$$


Since $\left(\widehat{A}: z_{n}\right)=\left(\cup_{i=2}^{n-3}\left\{x_{i} y_{i}, x_{i} y_{i+1}, x_{i} x_{i+1}, x_{i+1} y_{i}, y_{i} y_{i+1}, y_{i} z_{i}, y_{i} z_{i+1}, y_{i+1} z_{i}, z_{i} z_{i+1}\right\}\right.$,

$$
\left.x_{n-2} y_{n-2}, y_{n-2} z_{n-2}, z_{1}, y_{1}, z_{n-1}, y_{n-1}, y_{n}, x_{n}, x_{n-1} x_{n-2}, x_{n-1} y_{n-2}, x_{1} x_{2}, x_{1} y_{2}\right) \text {, }
$$

after renumbering the variables, we have $S_{n, 3} /\left(\widehat{A}: z_{n}\right) \cong\left(S_{n-3,3}^{\star \star} / I\left(\mathcal{P}_{n-3,3}^{\star \star}\right)\right)\left[z_{n}\right]$. Thus by Lemmas 3.5 and $2.7 \operatorname{depth}\left(S_{n, 3} /\left(\widehat{A}: z_{n}\right)\right)=\left\lceil\frac{n-3+2}{3}\right\rceil+1=\left\lceil\frac{n-1}{3}\right\rceil+1$. Also $S_{n, 3} /\left(\widehat{A}, z_{n}\right) \cong$ $S_{n-1,3} / I\left(\mathcal{P}_{n-1,3}\right)$. Therefore by Lemma $3.2 \operatorname{depth}\left(S_{n, 3} /\left(\widehat{A}, z_{n}\right)\right)=\left\lceil\frac{n-1}{3}\right\rceil$. By Remark 2.6

$$
\begin{aligned}
& \operatorname{depth}\left(S_{n, 3} / \widehat{A}\right) \geq \\
& \quad \min \left\{\operatorname{depth}\left(S_{n, 3} /\left(\widehat{A}: z_{n}\right)\right) \operatorname{depth}\left(S_{n, 3} /\left(\widehat{A}, z_{n}\right)\right)\right\}=\min \left\{\left\lceil\frac{n-1}{3}\right\rceil+1,\left\lceil\frac{n-1}{3}\right\rceil\right\}
\end{aligned}
$$

Hence combining Eq. 3.1, Eq. 3.2, Eq. 3.3 and Eq. 3.4 we get $\operatorname{depth}\left(S_{n, 3} / I\left(\mathcal{C}_{n, 3}\right)\right) \geq$ $\left\lceil\frac{n-1}{3}\right\rceil$. By Corollary 2.8 we have $\operatorname{depth}\left(S_{n, 3} / I\left(\mathcal{C}_{n, 3}\right)\right) \leq \operatorname{depth}\left(S_{n, 3} /\left(I\left(\mathcal{C}_{n, 3}\right): y_{n}\right)\right)$. Since $\left(S_{n, 3} /\left(I\left(\mathcal{C}_{n, 3}\right): y_{n}\right)\right) \cong\left(S_{n-3,3} /\left(I\left(\mathcal{P}_{n-3,3}\right)\right)\left[y_{n}\right]\right.$, by Lemmas 3.2 and 2.7 , we have $\operatorname{depth}\left(S_{n, 3} / I\left(\mathrm{e}_{n, 3}\right)\right) \leq\left\lceil\frac{n}{3}\right\rceil$, if $n \equiv 0(\bmod 3)$ or $n \equiv 2(\bmod 3)$ then $\left\lceil\frac{n-1}{3}\right\rceil=\left\lceil\frac{n}{3}\right\rceil$. If $n \equiv 1(\bmod 3)$ then $\left\lceil\frac{n-1}{3}\right\rceil \leq \operatorname{depth}\left(S_{n, 3} / I\left(\mathfrak{C}_{n, 3}\right)\right) \leq\left\lceil\frac{n}{3}\right\rceil$.

Now we prove the result for Stanley depth. If $n=3$, then by using [11] we have the following Stanley decomposition

$$
\begin{aligned}
& S_{3,3} / I\left(\mathcal{C}_{3,3}\right)=K\left[x_{1}\right] \oplus y_{1} K\left[y_{1}\right] \oplus z_{1} K\left[z_{1}\right] \oplus x_{2} K\left[x_{2}\right] \oplus y_{2} K\left[y_{2}\right] \oplus z_{2} K\left[z_{2}\right] \oplus \\
& \oplus x_{3} K\left[x_{3}\right] \oplus z_{3} K\left[z_{3}\right],
\end{aligned}
$$

$\operatorname{Thus} \operatorname{sdepth}\left(S_{3,3} / I\left(\mathcal{C}_{3,3}\right)\right) \geq 1$. For upper bound by Proposition 2.9 we have

$$
\operatorname{sdepth}\left(S_{3,3} / I\left(\mathcal{C}_{3,3}\right)\right) \leq \operatorname{sdepth}\left(S_{3,3} /\left(I\left(\mathcal{C}_{3,3}\right): y_{2}\right)\right),
$$

since $S_{3,3} /\left(I\left(\mathcal{C}_{3,3}\right): y_{2}\right) \cong K\left[y_{2}\right]$, therefore $\operatorname{sdepth}\left(S_{3,3} / I\left(\mathcal{C}_{3,3}\right)\right) \leq 1$, as desired. For $n=4$,

let $T:=K\left[x_{1}, z_{1}\right] \oplus y_{1} K\left[x_{3}, y_{1}\right] \oplus x_{2} K\left[x_{2}, z_{1}\right] \oplus y_{2} K\left[y_{2}, x_{4}\right] \oplus y_{3} K\left[x_{1}, y_{3}\right] \oplus x_{4} K\left[x_{4}, z_{1}\right]$

$$
\oplus y_{4} K\left[x_{2}, y_{4}\right] \oplus z_{4} K\left[x_{1}, z_{4}\right] \oplus z_{2} K\left[x_{1}, z_{2}\right] \oplus x_{3} K\left[x_{1}, x_{3}\right] \oplus z_{3} K\left[x_{1}, z_{3}\right],
$$

if $u \in S_{4,3} / I\left(\mathcal{C}_{4,3}\right)$ such that $u \notin T$, then $\operatorname{deg}\left(u_{i}\right) \geq 2$. It is easy to see that $S_{4,3} / I\left(\mathcal{C}_{4,3}\right)=$ $T \oplus_{u} u K[\operatorname{supp}(u)]$, Thus $\operatorname{sdepth}\left(S_{4,3} / I\left(\mathfrak{C}_{4,3}\right)\right) \geq 2$. For upper bound by Proposition 2.9 we have $\operatorname{sdepth}\left(S_{4,3} / I\left(\mathcal{C}_{4,3}\right)\right) \leq \operatorname{sdepth}\left(S_{4,3} /\left(I\left(\mathcal{C}_{4,3}\right): y_{2} y_{4}\right)\right)$, since $S_{4,3} /\left(I\left(\mathcal{C}_{4,3}\right): y_{2} y_{4}\right) \cong$ $K\left[y_{2}, y_{4}\right]$, therefore $\operatorname{sdepth}\left(S_{4,3} / I\left(\mathcal{C}_{4,3}\right)\right) \leq 2$. Hence $\operatorname{sdepth}\left(S_{4,3} / I\left(\mathcal{C}_{4,3}\right)\right)=2$. Let $n \geq 5$, using Proposition 2.9 instead of Corollary 2.8 the proof for depth also works for Stanley depth.

Example 3.7. One can expect that $\operatorname{depth}\left(S_{n, 3} / I\left(\mathcal{C}_{n, 3}\right)\right)=\left\lceil\frac{n-1}{3}\right\rceil$ as we have in $[4$, Proposition 1.3] and Theorem 3.3. But examples show that in the essential case when $n \equiv 1(\bmod 3)$ the upper bound in Theorem 3.6 is reached. For instance, when $n=4$, then $\operatorname{depth}\left(S_{4,3} / I\left(\mathfrak{C}_{4,3}\right)\right)=\operatorname{sdepth}\left(S_{4,3} / I\left(\mathfrak{C}_{4,3}\right)\right)=2=\left\lceil\frac{4}{3}\right\rceil$.

Remark 3.8. If $3 \leq n \leq 10$, then using SdepthLib:coc [25] we have $\operatorname{sdepth}\left(S_{n, 3} / I\left(\mathrm{C}_{n, 3}\right)\right)=\left\lceil\frac{n}{3}\right\rceil$. Also for $3 \leq n \leq 6$, we have $\operatorname{depth}\left(S_{n, 3} / I\left(\mathrm{C}_{n, 3}\right)\right)=\left\lceil\frac{n}{3}\right\rceil$ that is the upper bound in Theorem 3.6 is reached for both depth and Stanley depth in all known cases. In order to $\operatorname{show}$ that $\operatorname{sdepth}\left(S_{n, 3} / I\left(\mathcal{C}_{n, 3}\right)\right) \geq \operatorname{depth}\left(S_{n, 3} / I\left(\mathcal{C}_{n, 3}\right)\right)$ (Stanley's inequality) one needs to $\operatorname{show}$ that $\operatorname{sdepth}\left(S_{n, 3} / I\left(\mathcal{C}_{n, 3}\right)\right)=\left\lceil\frac{n}{3}\right\rceil$, for all $n$. For this one needs to find a suitable Stanley decomposition which we don't know at the moment and could be hard to find.

\section{Lower bounds for Stanley depth of $I\left(\mathcal{P}_{n, m}\right)$ and $I\left(\mathcal{C}_{n, m}\right)$ when $1 \leq m \leq 3$}

In this section, we give some lower bounds for Stanley depth of $I\left(\mathcal{P}_{n, m}\right)$ and $I\left(\mathfrak{C}_{n, m}\right)$, when $m \leq 3$. These bounds together with the results of the previous section allow us to give a positive answer to Conjecture 1 in some special cases. We begin this section with the following useful lemma: 
Lemma 4.1. Let $A$ and $B$ be two disjoint sets of variables, $I_{1} \subset K[A]$ and $I_{2} \subset K[B]$ be square free monomial ideals such that $\operatorname{sdepth}_{K[A]}\left(I_{1}\right)>\operatorname{sdepth}\left(K[A] / I_{1}\right)$. Then

$$
\operatorname{sdepth}_{K[A \cup B]}\left(I_{1}+I_{2}\right) \geq \operatorname{sdepth}\left(K[A] / I_{1}\right)+\operatorname{sdepth}_{K[B]}\left(I_{2}\right) .
$$

Proof. By [2, Theorem 1.3(1)] we have

$$
\operatorname{sdepth}_{K[A \cup B]}\left(I_{1}+I_{2}\right) \geq \min \left\{\operatorname{sdepth}_{K[A \cup B]}\left(I_{1}\right), \operatorname{sdepth}\left(K[A] / I_{1}\right)+\operatorname{sdepth}_{K[B]}\left(I_{2}\right)\right\} .
$$

Now by Lemma 2.7 we have

$$
\operatorname{sdepth}_{K[A \cup B]}\left(I_{1}+I_{2}\right) \geq \min \left\{\operatorname{sdepth}_{K[A]}\left(I_{1}\right)+|B|, \operatorname{sdepth}\left(K[A] / I_{1}\right)+\operatorname{sdepth}_{K[B]}\left(I_{2}\right)\right\} .
$$

Since $|B| \geq \operatorname{sdepth}_{K[B]}\left(I_{2}\right)$, therefore

$$
\operatorname{sdepth}_{K[A]}\left(I_{1}\right)+|B|>\operatorname{sdepth}\left(K[A] / I_{1}\right)+\operatorname{sdepth}_{K[B]}\left(I_{2}\right),
$$

this proves the desired inequality.

Now we introduce some notations for the case $m=3$. For $3 \leq l \leq n-2$, let

$$
\begin{aligned}
J_{l}:=\left(x_{n-l}, z_{n-l}, x_{n-l+1}, y_{n-l-1}, z_{n-l+1}, x_{n-l-1}, z_{n-l-1}\right), \\
I\left(P_{l-1}^{\prime}\right):=\left(x_{n-l+2} x_{n-l+3}, \ldots, x_{n-1} x_{n}\right), \\
I\left(P_{l-1}^{\prime \prime}\right):=\left(z_{n-l+2} z_{n-l+3}, \ldots, z_{n-1} z_{n}\right),
\end{aligned}
$$

be the monomial ideals of $S_{n, 3}$. Consider the subsets of variables

$$
\begin{gathered}
D_{l}:=\left\{x_{n-l+2}, x_{n-l+3}, \ldots, x_{n-1}, x_{n}\right\}, \\
D_{l}^{\prime}:=\left\{z_{n-l+2}, z_{n-l+3}, \ldots, z_{n-1}, z_{n}\right\}, \\
D_{l}^{\prime \prime}:=\left\{x_{n-l}, z_{n-l}, x_{n-l+1}, y_{n-l-1}, z_{n-l+1}, x_{n-l-1}, z_{n-l-1}\right\} .
\end{gathered}
$$

Let $L_{l}$ be a monomial ideal of $S_{n, 3}$ such that $L_{l}=I\left(P_{l-1}^{\prime}\right)+I\left(P_{l-1}^{\prime \prime}\right)+J_{l}$. With these notations we have the following lemma:

Lemma 4.2. For $3 \leq l \leq n-2, \operatorname{sdepth}_{K\left[D_{l} \cup D_{l}^{\prime} \cup D_{l}^{\prime \prime}\right]}\left(L_{l}\right) \geq\left\lceil\frac{l+2}{3}\right\rceil+1$.

Proof. Since $L_{l}=I\left(P_{l-1}^{\prime}\right)+I\left(P_{l-1}^{\prime \prime}\right)+J_{l}$, by [2, Theorem 1.3], we have

$$
\begin{aligned}
\operatorname{sdepth}_{K\left[D_{l} \cup D_{l}^{\prime} \cup D_{l}^{\prime \prime}\right]}\left(L_{l}\right) \geq & \min \left\{\operatorname{sdepth}_{K\left[D_{l} \cup D_{l}^{\prime} \cup D_{l}^{\prime \prime}\right]}\left(J_{l}\right), \min \left\{\operatorname{sdepth}_{K\left[D_{l} \cup D_{l}^{\prime}\right]}\left(I\left(P_{l-1}^{\prime}\right)\right),\right.\right. \\
& \left.\left.\operatorname{sdepth}_{K\left[D_{l}\right]}\left(K\left[D_{l}\right] / I\left(P_{l-1}^{\prime}\right)\right)+\operatorname{sdepth}_{K\left[D_{l}^{\prime}\right]}\left(I\left(P_{l-1}^{\prime \prime}\right)\right)\right\}\right\} .
\end{aligned}
$$

By using [21, Theorem 2.3] and [22, Proposition 2.1], Eq. 4.1 implies that

$$
\begin{aligned}
\operatorname{sdepth}_{K\left[D_{l} \cup D_{l}^{\prime} \cup D_{l}^{\prime \prime}\right]}\left(L_{l}\right) & \geq \min \left\{4+2(l-2), \min \left\{2 l-2-\left\lfloor\frac{l-2}{2}\right\rfloor,\left\lceil\frac{l-1}{3}\right\rceil+l-1-\left\lfloor\frac{l-2}{2}\right\rfloor\right\}\right\} \\
& \geq\left\lceil\frac{l+2}{3}\right\rceil+1 .
\end{aligned}
$$

Theorem 4.3. For $n \geq 1$ and $1 \leq m \leq 3$,

$$
\operatorname{sdepth}\left(I\left(\mathcal{P}_{n, m}\right)\right)>\operatorname{sdepth}\left(S_{n, m} / I\left(\mathcal{P}_{n, m}\right)\right)=\left\lceil\frac{n}{3}\right\rceil .
$$

Proof. By Lemma 3.2 and Remark 3.1 we have $\operatorname{sdepth}\left(S_{n, m} / I\left(\mathcal{P}_{n, m}\right)\right)=\left\lceil\frac{n}{3}\right\rceil$, we use this fact frequently in the proof without referring it again and again.

(a) If $m=1$, clearly $I\left(\mathcal{P}_{n, 1}\right) \cong I\left(P_{n}\right)$, thus by [21, Theorem 2.3] and [22, Proposition 2.1] we have $\operatorname{sdepth}\left(I\left(\mathcal{P}_{n, 1}\right)\right)>\operatorname{sdepth}\left(S_{n, 1} / I\left(\mathcal{P}_{n, 1}\right)\right)=\left\lceil\frac{n}{3}\right\rceil$. 
(b) If $m=2$, we prove the result by induction on $n$. If $n=1$ then by (a) the required result follows. If $n=2,3$, then by [19, Lemma 2.1], $\operatorname{sdepth}\left(I\left(\mathcal{P}_{n, 2}\right)\right)>\left\lceil\frac{n}{3}\right\rceil$. Now assume that $n \geq 4$. Since $x_{n-1} \notin I\left(\mathcal{P}_{n, 2}\right)$, thus we have

$$
I\left(\mathcal{P}_{n, 2}\right)=I\left(\mathcal{P}_{n, 2}\right) \cap S^{\prime} \oplus x_{n-1}\left(I\left(\mathcal{P}_{n, 2}\right): x_{n-1}\right) S_{n, 2},
$$

where $S^{\prime}=K\left[x_{1}, x_{2}, \ldots, x_{n-2}, x_{n}, y_{1}, y_{2}, \ldots, y_{n}\right]$. Now

$I\left(\mathcal{P}_{n, 2}\right) \cap S^{\prime}=\left(\mathcal{G}\left(I\left(\mathcal{P}_{n-2,2}\right)\right), x_{n-2} y_{n-1}, y_{n-2} y_{n-1}, x_{n} y_{n}, y_{n-1} x_{n}, y_{n-1} y_{n}\right)$ and

$$
\left(I\left(\mathcal{P}_{n, 2}\right): x_{n-1}\right) S_{n, 2}=\left(\mathcal{G}\left(I\left(\mathcal{P}_{n-3,2}\right)\right), x_{n-2}, y_{n-2}, y_{n-1}, x_{n}, y_{n}\right) S_{n, 2} .
$$

As $y_{n-1} \notin I\left(\mathcal{P}_{n, 2}\right) \cap S^{\prime}$, so we get

$$
I\left(\mathcal{P}_{n, 2}\right) \cap S^{\prime}=\left(I\left(\mathcal{P}_{n, 2}\right) \cap S^{\prime}\right) \cap S^{\prime \prime} \oplus y_{n-1}\left(I\left(\mathcal{P}_{n, 2}\right) \cap S^{\prime}: y_{n-1}\right) S^{\prime},
$$

where $S^{\prime \prime}=K\left[x_{1}, \ldots, x_{n-2}, x_{n}, y_{1}, \ldots, y_{n-2}, y_{n}\right]$. Thus

$I\left(\mathcal{P}_{n, 2}\right)=\left(I\left(\mathcal{P}_{n, 2}\right) \cap S^{\prime}\right) \cap S^{\prime \prime} \oplus y_{n-1}\left(I\left(\mathcal{P}_{n, 2}\right) \cap S^{\prime}: y_{n-1}\right) S^{\prime} \oplus x_{n-1}\left(I\left(\mathcal{P}_{n, 2}\right): x_{n-1}\right) S_{n, 2}$,

where

$$
\left(I\left(\mathcal{P}_{n, 2}\right) \cap S^{\prime}\right) \cap S^{\prime \prime}=\left(\mathcal{G}\left(I\left(\mathcal{P}_{n-2,2}\right)\right), x_{n} y_{n}\right) S^{\prime \prime}
$$

and

$$
\left(I\left(\mathcal{P}_{n, 2}\right) \cap S^{\prime}: y_{n-1}\right) S^{\prime}=\left(\mathcal{G}\left(I\left(\mathcal{P}_{n-3,2}\right)\right), x_{n-2}, y_{n-2}, x_{n}, y_{n}\right) S^{\prime} .
$$

By induction on $n$ and Lemma 4.1 we have $\operatorname{sdepth}\left(\left(I\left(\mathcal{P}_{n, 2}\right) \cap S^{\prime}\right) \cap S^{\prime \prime}\right) \geq \operatorname{sdepth}\left(S_{n-2,2} / I\left(\mathcal{P}_{n-2,2}\right)\right)+\operatorname{sdepth}_{K\left[x_{n}, y_{n}\right]}\left(x_{n} y_{n}\right)$.

Again by induction on $n$, Lemma 4.1 and Lemma 2.7 we have

$\operatorname{sdepth}\left(\left(I\left(\mathcal{P}_{n, 2}\right) \cap S^{\prime}: y_{n-1}\right) S^{\prime}\right) \geq \operatorname{sdepth}\left(S_{n-3,2} / I\left(\mathcal{P}_{n-3,2}\right)\right)+\operatorname{sdepth}_{T}\left(x_{n-2}, y_{n-2}, x_{n}, y_{n}\right)+1$

and

$\operatorname{sdepth}\left(\left(I\left(\mathcal{P}_{n, 2}\right): x_{n-1}\right) S_{n, 2}\right) \geq$

$$
\operatorname{sdepth}\left(S_{n-3,2} / I\left(\mathcal{P}_{n-3,2}\right)\right)+\operatorname{sdepth}_{R}\left(x_{n-2}, y_{n-2}, y_{n-1}, x_{n}, y_{n}\right)+1,
$$

where $T=\left[x_{n-2}, y_{n-2}, x_{n}, y_{n}\right]$ and $R=K\left[x_{n-2}, y_{n-2}, y_{n-1}, x_{n}, y_{n}\right]$. Thus

$$
\operatorname{sdepth}\left(\left(I\left(\mathcal{P}_{n, 2}\right) \cap S^{\prime}\right) \cap S^{\prime \prime}\right)>\left\lceil\frac{n}{3}\right\rceil
$$

as $\operatorname{sdepth}_{K\left[x_{n}, y_{n}\right]}\left(x_{n} y_{n}\right)=2$. By [1, Theorem 2.2] we have $\operatorname{sdepth}\left(\left(I\left(\mathcal{P}_{n, 2}\right) \cap S^{\prime}\right.\right.$ : $\left.\left.y_{n-1}\right) S^{\prime}\right)>\left\lceil\frac{n}{3}\right\rceil$ and $\operatorname{sdepth}\left(\left(I\left(\mathcal{P}_{n, 2}\right): x_{n-1}\right) S_{n, 2}\right)>\left\lceil\frac{n}{3}\right\rceil$. This completes the proof for $m=2$.

(c) If $m=3$, we proceed again by induction on $n$. If $n=1$, then by (a) the required result follows. If $n=2$, the result follows by (b). If $n=3$ then by [19, Lemma 2.1] $\operatorname{sdepth}\left(I\left(\mathcal{P}_{3,3}\right)\right)>\left\lceil\frac{3}{3}\right\rceil$. If $n \geq 4$, then we consider the following decomposition of $I\left(\mathcal{P}_{n, 3}\right)$ as a vector space:

$$
I\left(\mathcal{P}_{n, 3}\right)=I\left(\mathcal{P}_{n, 3}\right) \cap R_{1} \oplus y_{n}\left(I\left(\mathcal{P}_{n, 3}\right): y_{n}\right) S_{n, 3} .
$$

Similarly, we can decompose $I\left(\mathcal{P}_{n, 3}\right) \cap R_{1}$ by the following:

$$
I\left(\mathcal{P}_{n, 3}\right) \cap R_{1}=I\left(\mathcal{P}_{n, 3}\right) \cap R_{2} \oplus y_{n-1}\left(I\left(\mathcal{P}_{n, 3}\right) \cap R_{1}: y_{n-1}\right) R_{1} .
$$

Continuing in the same way for $1 \leq l \leq n-1$ we have

$$
I\left(\mathcal{P}_{n, 3}\right) \cap R_{l}=I\left(\mathcal{P}_{n, 3}\right) \cap R_{l+1} \oplus y_{n-l}\left(I\left(\mathcal{P}_{n, 3}\right) \cap R_{l}: y_{n-l}\right) R_{l},
$$

where $R_{l}:=K\left[x_{1}, x_{2}, \ldots x_{n}, y_{1}, y_{2}, \ldots, y_{n-l}, z_{1}, z_{2}, \ldots, z_{n}\right]$. Finally, we get the following decomposition of $I\left(\mathcal{P}_{n, 3}\right)$ :

$$
I\left(\mathcal{P}_{n, 3}\right)=I\left(\mathcal{P}_{n, 3}\right) \cap R_{n} \oplus \oplus_{l=1}^{n-1} y_{n-l}\left(I\left(\mathcal{P}_{n, 3}\right) \cap R_{l}: y_{n-l}\right) R_{l} \oplus y_{n}\left(I\left(\mathcal{P}_{n, 3}\right): y_{n}\right) S_{n, 3} .
$$


Therefore

$\operatorname{sdepth}\left(I\left(\mathcal{P}_{n, 3}\right)\right) \geq \min \left\{\operatorname{sdepth}\left(I\left(\mathcal{P}_{n, 3}\right) \cap R_{n}\right), \operatorname{sdepth}\left(\left(I\left(\mathcal{P}_{n, 3}\right): y_{n}\right) S_{n, 3}\right)\right.$,

$$
\left.\min _{l=1}^{n-1}\left\{\operatorname{sdepth}\left(\left(I\left(\mathcal{P}_{n, 3}\right) \cap R_{l}: y_{n-l}\right) R_{l}\right)\right\}\right\} \text {. }
$$

Since

$I\left(\mathcal{P}_{n, 3}\right) \cap R_{n}=\left(\left(x_{1} x_{2}, x_{2} x_{3}, \ldots, x_{n-1} x_{n}\right)+\left(z_{1} z_{2}, z_{2} z_{3}, \ldots, z_{n-1} z_{n}\right)\right) K\left[x_{1}, \ldots, x_{n}, z_{1}, \ldots, z_{n}\right]$,

thus by [2, Theorem 1.3] and [22, Proposition 2.1] we have $\operatorname{sdepth}\left(I\left(\mathcal{P}_{n, 3}\right) \cap R_{n}\right)>$

$\left\lceil\frac{n}{3}\right\rceil$. As we can see that

$$
\left(I\left(\mathcal{P}_{n, 3}\right): y_{n}\right) S_{n, 3}=\left(\mathcal{G}\left(I\left(\mathcal{P}_{n-2,3}\right)\right)+\left(x_{n}, z_{n}, x_{n-1}, z_{n-1}, y_{n-1}\right)\right)\left[y_{n}\right] .
$$

Let $B:=K\left[x_{n}, z_{n}, x_{n-1}, z_{n-1}, y_{n-1}\right]$ thus by induction on $n$, Lemmas 4.1 and 2.7 $\operatorname{sdepth}\left(\left(I\left(\mathcal{P}_{n, 3}\right): y_{n}\right) S_{n, 3}\right)>\operatorname{sdepth}\left(S_{n-2,3} / I\left(\mathcal{P}_{n-2,3}\right)\right)+\operatorname{sdepth}_{B}\left(x_{n}, z_{n}, x_{n-1}, z_{n-1}, y_{n-1}\right)+1$.

By $\left[1\right.$, Theorem 2.2] we have $\operatorname{sdepth}\left(\left(I\left(\mathcal{P}_{n, 3}\right): y_{n}\right) S_{n, 3}\right)>\left\lceil\frac{n}{3}\right\rceil$.

(1): If $l=1$, then $\left(I\left(\mathcal{P}_{n, 3}\right) \cap R_{1}: y_{n-1}\right) R_{1}=\left(\mathcal{G}\left(I\left(\mathcal{P}_{n-3,3}\right)\right)+J_{1}\right)\left[y_{n-1}\right]$, where $J_{1}:=\left(x_{n-1}, z_{n-1}, x_{n}, y_{n-2}, z_{n}, x_{n-2}, z_{n-2}\right)$, then by induction on $n$, Lemmas 4.1 and 2.7 , we have

$\operatorname{sdepth}\left(\left(I\left(\mathcal{P}_{n, 3}\right) \cap R_{1}: y_{n-1}\right) R_{1}\right)>\operatorname{sdepth}\left(S_{n-3,3} / I\left(\mathcal{P}_{n-3,3}\right)\right)+\operatorname{sdepth}_{K\left[\operatorname{supp}\left(J_{1}\right)\right]}\left(J_{1}\right)+1$,

by $\left[1\right.$, Theorem 2.2] we have $\operatorname{sdepth}\left(\left(I\left(\mathcal{P}_{n, 3}\right) \cap R_{1}: y_{n-1}\right) R_{1}\right)>\left\lceil\frac{n}{3}\right\rceil$.

(2): If $l=2$ and $n \neq 4$, then

$$
\left(I\left(\mathcal{P}_{n, 3}\right) \cap R_{2}: y_{n-2}\right) R_{2}=\left(\mathcal{G}\left(I\left(\mathcal{P}_{n-4,3}\right)\right)+J_{2}\right)\left[y_{n-2}, x_{n}, z_{n}\right],
$$

where $J_{2}:=\left(x_{n-2}, z_{n-2}, x_{n-1}, z_{n-1}, x_{n-3}, y_{n-3}, z_{n-3}\right)$, using the same arguments as in case(1) we have $\operatorname{sdepth}\left(\left(I\left(\mathcal{P}_{n, 3}\right) \cap R_{2}: y_{n-2}\right) R_{2}\right)>\left\lceil\frac{n}{3}\right\rceil$.

(3): If $3 \leq l \leq n-3$, then $\left(I\left(\mathcal{P}_{n, 3}\right) \cap R_{l}: y_{n-l}\right) R_{l}=\left(\mathcal{G}\left(I\left(\mathcal{P}_{n-(l+2), 3}\right)\right)+\right.$ $\left.\mathcal{G}\left(L_{l}\right)\right)\left[y_{n-l}\right]$, by induction on $n$, Lemmas 4.1 and 2.7 , we have

$$
\begin{aligned}
\operatorname{sdepth}\left(\left(I\left(\mathcal{P}_{n, 3}\right) \cap R_{l}: y_{n-l}\right) R_{l}\right)> & \operatorname{sdepth}\left(S_{n-(l+2), 3} /\left(I\left(\mathcal{P}_{n-(l+2), 3}\right)\right)\right) \\
& +\operatorname{sdepth}_{K\left[D_{l} \cup D_{l}^{\prime} \cup D_{l}^{\prime \prime}\right]}\left(L_{l}\right)+1,
\end{aligned}
$$

By Eq. 4.3 and Lemma 4.2 we have

$\operatorname{sdepth}\left(\left(I\left(\mathcal{P}_{n, 3}\right) \cap R_{l}: y_{n-l}\right) R_{l}\right)>\left\lceil\frac{n-(l+2)}{3}\right\rceil+\left\lceil\frac{l+2}{3}\right\rceil+1+1>\left\lceil\frac{n}{3}\right\rceil$.

(4): If $l=n-2$, then $\left(I\left(\mathcal{P}_{n, 3}\right) \cap R_{n-2}: y_{2}\right) R_{n-2}=\left(\mathcal{G}\left(L_{n-2}\right)\right)\left[y_{2}\right]$, by Lemmas 4.2 and 2.7 we have $\operatorname{sdepth}\left(\left(I\left(\mathcal{P}_{n, 3}\right) \cap R_{n-2}: y_{2}\right) R_{n-2}\right)>\left\lceil\frac{n}{3}\right\rceil$.

(5): If $l=n-1$, then

$\left(I\left(\mathcal{P}_{n, 3}\right) \cap R_{n-1}: y_{1}\right) R_{n-1}=\left(I\left(P_{n-2}^{\prime}\right)+I\left(P_{n-2}^{\prime \prime}\right)+J_{n-1}\right) K\left[D_{n-1} \cup D_{n-1}^{\prime} \cup D_{n-1}^{\prime \prime} \cup\left\{y_{1}\right\}\right]$, where $\mathcal{G}\left(J_{n-1}\right)=\left\{x_{1}, z_{1}, x_{2}, z_{2}\right\}, \quad D_{n-1}=\left\{x_{3}, x_{4}, \ldots, x_{n}\right\}, D_{n-1}^{\prime}=$ $\left\{z_{3}, z_{4}, \ldots, z_{n}\right\}$ and $D_{n-1}^{\prime \prime}=\left\{x_{1}, z_{1}, x_{2}, z_{2}\right\}$. Using the proof of Lemma 4.2 and by Lemma 2.7

$\operatorname{sdepth}_{K\left[D_{n-1} \cup D_{n-1}^{\prime} \cup D_{n-1}^{\prime \prime} \cup\left\{y_{1}\right\}\right]}\left(I\left(P_{n-2}^{\prime}\right)+I\left(P_{n-2}^{\prime \prime}\right)+J_{n-1}\right)>\left\lceil\frac{n}{3}\right\rceil$,

that is $\operatorname{sdepth}\left(\left(I\left(\mathcal{P}_{n, 3}\right) \cap R_{n-1}: y_{1}\right) R_{n-1}\right)>\left\lceil\frac{n}{3}\right\rceil$.

Thus by Eq. 4.2 we get $\operatorname{sdepth}\left(I\left(\mathcal{P}_{n, 3}\right)\right)>\left\lceil\frac{n}{3}\right\rceil$. 
Proposition 4.4. For $n \geq 3, \operatorname{sdepth}\left(I\left(\mathcal{C}_{n, 2}\right) / I\left(\mathcal{P}_{n, 2}\right)\right) \geq\left\lceil\frac{n+2}{3}\right\rceil$.

Proof. For $3 \leq n \leq 5$, we use [11] to show that there exist Stanley decompositions of desired Stanley depth. When $n=3$ or 4 , then

$$
I\left(\mathfrak{C}_{n, 2}\right) / I\left(\mathcal{P}_{n, 2}\right)=x_{1} x_{n} K\left[x_{1}, x_{n}\right] \oplus x_{1} y_{n} K\left[x_{1}, y_{n}\right] \oplus y_{1} x_{n} K\left[y_{1}, x_{n}\right] \oplus y_{1} y_{n} K\left[y_{1}, y_{n}\right] .
$$

If $n=5$, then

$I\left(\mathcal{C}_{5,2}\right) / I\left(\mathcal{P}_{5,2}\right)=x_{1} x_{5} K\left[x_{1}, x_{3}, x_{5}\right] \oplus x_{1} y_{5} K\left[x_{1}, x_{3}, y_{5}\right] \oplus y_{1} x_{5} K\left[y_{1}, x_{3}, x_{5}\right] \oplus y_{1} y_{5} K\left[y_{1}, x_{3}, y_{5}\right]$ $\oplus x_{1} y_{3} x_{5} K\left[x_{1}, y_{3}, x_{5}\right] \oplus x_{1} y_{3} y_{5} K\left[x_{1}, y_{3}, y_{5}\right] \oplus y_{1} y_{3} y_{5} K\left[y_{1}, y_{3}, y_{5}\right] \oplus y_{1} y_{3} x_{5} K\left[y_{1}, y_{3}, x_{5}\right]$.

Let $n \geq 6$ and $T:=\left(\bigcup_{i=3}^{n-3}\left\{x_{i} y_{i}, x_{i} y_{i+1}, x_{i} x_{i+1}, x_{i+1} y_{i}, y_{i} y_{i+1}\right\}, x_{n-2} y_{n-2}\right) \subset \tilde{S}$, where $\tilde{S}:=K\left[x_{3}, x_{4}, \ldots, x_{n-2}, y_{3}, y_{4} \ldots, y_{n-2}\right]$. Then we have the following $K$-vector space isomorphism:

$$
I\left(\mathcal{C}_{n, 2}\right) / I\left(\mathcal{P}_{n, 2}\right) \cong x_{1} x_{n} \frac{\tilde{S}}{T}\left[x_{1}, x_{n}\right] \oplus y_{1} y_{n} \frac{\tilde{S}}{T}\left[y_{1}, y_{n}\right] \oplus x_{1} y_{n} \frac{\tilde{S}}{T}\left[x_{1}, y_{n}\right] \oplus y_{1} x_{n} \frac{\tilde{S}}{T}\left[y_{1}, x_{n}\right] .
$$

Thus by Lemmas 3.2 and 2.7 , we have $\operatorname{sdepth}\left(I\left(\mathcal{C}_{n, 2}\right) / I\left(\mathcal{P}_{n, 2}\right)\right) \geq\left\lceil\frac{n+2}{3}\right\rceil$.

For $n \geq 6$, let $Q=\left\{x_{1}, y_{1}, x_{2}, y_{2}, x_{n}, y_{n}, x_{n-1}, y_{n-1}\right\}$. Consider a subgraph $\mathcal{C}_{n, 3}^{\diamond}$ of $\mathcal{C}_{n, 3}$ with vertex set $V\left(\mathfrak{C}_{n, 3}^{\diamond}\right)=V\left(\mathfrak{C}_{n, 3}\right) \backslash Q$ and edge set

$$
E\left(\mathfrak{C}_{n, 3}^{\diamond}\right)=E\left(\mathfrak{C}_{n, 3}\right) \backslash\left\{e \in E\left(\mathcal{C}_{n, 3}\right): \text { where } e \text { has at least one end vertex in } Q\right\} .
$$

For example of $\mathcal{C}_{n, 3}^{\diamond}$ see Fig. 5 .

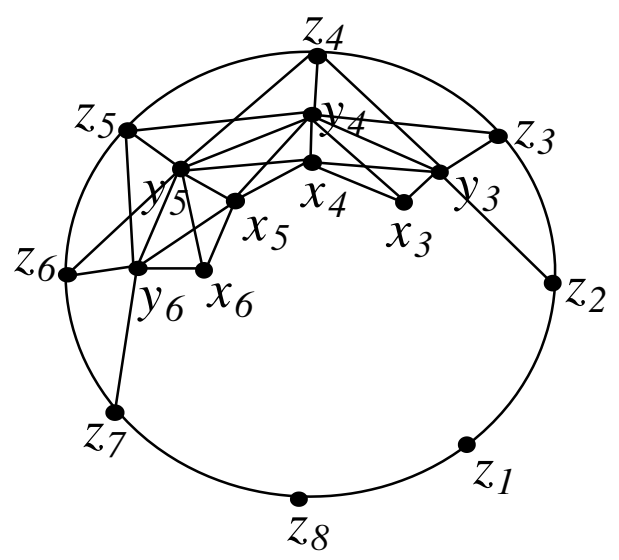

Figure 5. $\mathrm{C}_{8,3}^{\diamond}$.

Lemma 4.5. Let $n \geq 6$, if $n \equiv 0(\bmod 3)$, then $\operatorname{sdepth}\left(S_{n, 3}^{\diamond} / I\left(C_{n, 3}^{\diamond}\right)\right)=\left\lceil\frac{n-2}{3}\right\rceil$. Otherwise, $\left\lceil\frac{n-2}{3}\right\rceil \leq \operatorname{sdepth}\left(S_{n, 3}^{\diamond} / I\left(C_{n, 3}^{\diamond}\right)\right) \leq\left\lceil\frac{n}{3}\right\rceil$.

Proof. By Remark 2.6

$\operatorname{sdepth}\left(S_{n, 3}^{\diamond} / I\left(C_{n, 3}^{\diamond}\right)\right) \geq \min \left\{\operatorname{sdepth}\left(S_{n, 3}^{\diamond} /\left(I\left(C_{n, 3}^{\diamond}\right): z_{1}\right)\right), \operatorname{sdepth}\left(S_{n, 3}^{\diamond} /\left(I\left(C_{n, 3}^{\diamond}\right), z_{1}\right)\right)\right\}$.

Since $\left(I\left(C_{n, 3}^{\diamond}\right): z_{1}\right)=\left(\left(\cup_{i=3}^{n-3}\left\{x_{i} y_{i}, x_{i} y_{i+1}, x_{i} x_{i+1}, x_{i+1} y_{i}, y_{i} y_{i+1}, y_{i} z_{i}, y_{i} z_{i+1}, y_{i+1} z_{i}, z_{i} z_{i+1}\right\}\right.\right.$,

$$
\left.\left.x_{n-2} y_{n-2}, y_{n-2} z_{n-2}\right), y_{n-2} z_{n-1}, z_{n-2} z_{n-1}, z_{2}, z_{n}\right) \text {, }
$$

so after renumbering the variables we have $S_{n, 3}^{\diamond} /\left(I\left(C_{n, 3}^{\diamond}\right): z_{1}\right) \cong S_{n-4,3}^{\star} / I\left(\mathcal{P}_{n-4,3}^{\star}\right)\left[z_{1}\right]$. Therefore, by Lemmas 2.7 and 3.5,

$$
\operatorname{sdepth}\left(S_{n, 3}^{\diamond} /\left(I\left(C_{n, 3}^{\diamond}\right): z_{1}\right)\right)=\left\lceil\frac{n-4+1}{3}\right\rceil+1=\left\lceil\frac{n}{3}\right\rceil .
$$


Now let

$$
\begin{aligned}
B:=\left(I\left(C_{n, 3}^{\diamond}\right), z_{1}\right)= & \left(\left(\cup_{i=3}^{n-3}\left\{x_{i} y_{i}, x_{i} y_{i+1}, x_{i} x_{i+1}, x_{i+1} y_{i}, y_{i} y_{i+1}, y_{i} z_{i}, y_{i} z_{i+1}, y_{i+1} z_{i}, z_{i} z_{i+1}\right\},\right.\right. \\
& \left.\left.x_{n-2} y_{n-2}, y_{n-2} z_{n-2}\right), y_{n-2} z_{n-1}, z_{n-2} z_{n-1}, z_{n-1} z_{n}, y_{3} z_{2}, z_{2} z_{3}, z_{1}\right),
\end{aligned}
$$

so by Remark 2.6

$$
\operatorname{sdepth}\left(S_{n, 3}^{\diamond} / B\right) \geq \min \left\{\operatorname{sdepth}\left(S_{n, 3}^{\diamond} /\left(B: z_{n}\right)\right), \operatorname{sdepth}\left(S_{n, 3}^{\diamond} /\left(B, z_{n}\right)\right)\right\} .
$$

Since

$$
\begin{array}{r}
\left(B: z_{n}\right)=\left(\left(\cup_{i=3}^{n-3}\left\{x_{i} y_{i}, x_{i} y_{i+1}, x_{i} x_{i+1}, x_{i+1} y_{i}, y_{i} y_{i+1}, y_{i} z_{i}, y_{i} z_{i+1}, y_{i+1} z_{i}, z_{i} z_{i+1}\right\},\right.\right. \\
\left.\left.x_{n-2} y_{n-2}, y_{n-2} z_{n-2}\right), y_{3} z_{2}, z_{2} z_{3}, z_{1}, z_{n-1}\right),
\end{array}
$$

after renumbering the variables we have $S_{n, 3}^{\diamond} /\left(B: z_{n}\right) \cong S_{n-4,3}^{\star} / I\left(\mathcal{P}_{n-4,3}^{\star}\right)\left[z_{n}\right]$. Therefore by Lemmas 2.7 and $3.5, \operatorname{sdepth}\left(S_{n, 3}^{\diamond} /\left(B: z_{n}\right)\right)=\left\lceil\frac{n-4+1}{3}\right\rceil+1=\left\lceil\frac{n}{3}\right\rceil$. Now

$$
\begin{array}{r}
\left(B, z_{n}\right)=\left(\left(\bigcup_{i=3}^{n-3}\left\{x_{i} y_{i}, x_{i} y_{i+1}, x_{i} x_{i+1}, x_{i+1} y_{i}, y_{i} y_{i+1}, y_{i} z_{i}, y_{i} z_{i+1}, y_{i+1} z_{i}, z_{i} z_{i+1}\right\},\right.\right. \\
\left.\left.x_{n-2} y_{n-2}, y_{n-2} z_{n-2}\right), y_{n-2} z_{n-1}, z_{n-2} z_{n-1}, y_{3} z_{2}, z_{2} z_{3}, z_{1}, z_{n}\right),
\end{array}
$$

after renumbering the variables we have $S_{n, 3}^{\diamond} /\left(B, z_{n}\right) \cong S_{n-4,3}^{\star \star} / I\left(\mathcal{P}_{n-4,3}^{\star \star}\right)$. Therefore by Lemma 3.5, we have

$$
\operatorname{sdepth}\left(S_{n, 3}^{\diamond} /\left(B, z_{n}\right)\right)=\left\lceil\frac{n-4+2}{3}\right\rceil=\left\lceil\frac{n-2}{3}\right\rceil .
$$

Combining Eq. 4.4 and Eq. 4.5 we get $\left\lceil\frac{n-2}{3}\right\rceil \leq \operatorname{sdepth}\left(S_{n, 3}^{\diamond} / I\left(C_{n, 3}^{\diamond}\right)\right)$. For upper bound, as $z_{1} \notin I\left(C_{n, 3}^{\diamond}\right)$ so by Proposition 2.9

$$
\operatorname{sdepth}\left(S_{n, 3}^{\diamond} / I\left(C_{n, 3}^{\diamond}\right)\right) \leq \operatorname{sdepth}\left(S_{n, 3}^{\diamond} /\left(I\left(C_{n, 3}^{\diamond}\right): z_{1}\right)\right) .
$$

Since $\left(S_{n, 3}^{\diamond} /\left(I\left(C_{n, 3}^{\diamond}\right): z_{1}\right)\right) \cong\left(S_{n-4,3}^{\star} / I\left(\mathcal{P}_{n-4,3}^{\star}\right)\right)\left[z_{1}\right]$. Thus by Lemmas 2.7 and 3.5 ,

$$
\operatorname{sdepth}\left(S_{n, 3}^{\diamond} / I\left(C_{n, 3}^{\diamond}\right)\right) \leq\left\lceil\frac{n}{3}\right\rceil
$$

if $n \equiv 0(\bmod 3)$ then $\left\lceil\frac{n-2}{3}\right\rceil=\left\lceil\frac{n}{3}\right\rceil$. If $n \equiv 1(\bmod 3)$ or $n \equiv 2(\bmod 3)$ then

$$
\left\lceil\frac{n-2}{3}\right\rceil \leq \operatorname{sdepth}\left(S_{n, 3}^{\diamond} / I\left(C_{n, 3}^{\diamond}\right)\right) \leq\left\lceil\frac{n}{3}\right\rceil .
$$

Proposition 4.6. For $n \geq 3, \operatorname{sdepth}\left(I\left(\mathcal{C}_{n, 3}\right) / I\left(\mathcal{P}_{n, 3}\right)\right) \geq\left\lceil\frac{n+2}{3}\right\rceil$.

Proof. For $3 \leq n \leq 4$, as the minimal generators of $I\left(\mathcal{C}_{n, 3}\right) / I\left(\mathcal{P}_{n, 3}\right)$ have degree 2 , so by $[19, \operatorname{Lemma} 2.1] \operatorname{sdepth}\left(I\left(\mathcal{C}_{n, 3}\right) / I\left(\mathcal{P}_{n, 3}\right)\right) \geq 2=\left\lceil\frac{n+2}{3}\right\rceil$. If $n=5$ then we use [11] to show that there exist Stanley decompositions of desired Stanley depth. Let

$$
\begin{array}{r}
H:=x_{1} x_{5} K\left[x_{1}, x_{3}, x_{5}\right] \oplus x_{1} y_{5} K\left[x_{1}, x_{3}, y_{5}\right] \oplus y_{1} x_{5} K\left[x_{3}, x_{5}, y_{1}\right] \oplus y_{1} y_{5} K\left[x_{3}, y_{1}, y_{5}\right] \\
\oplus z_{1} y_{5} K\left[x_{3}, y_{5}, z_{1}\right] \oplus z_{1} z_{5} K\left[z_{1}, z_{3}, z_{5}\right] \oplus y_{1} z_{5} K\left[y_{1}, y_{3}, z_{5}\right]
\end{array}
$$

Clearly, $H \subset I\left(\mathcal{C}_{5,3}\right) / I\left(\mathcal{P}_{5,3}\right)$. Let $v \in I\left(\mathcal{C}_{5,3}\right) / I\left(\mathcal{P}_{5,3}\right)$ be a sqaurefree monomial such that $v \notin H$ then $\operatorname{deg}(v) \geq 3$. Since

$$
I\left(\mathcal{C}_{5,3}\right) / I\left(\mathcal{P}_{5,3}\right)=H \oplus_{v} v K[\operatorname{supp}(v)],
$$

thus we have $\operatorname{sdepth}\left(I\left(\mathcal{C}_{5,3}\right) / I\left(\mathcal{P}_{5,3}\right)\right) \geq 3=\left\lceil\frac{5+2}{3}\right\rceil$. Now for $n \geq 6$, let $U:=\left(\cup_{i=3}^{n-3}\left\{x_{i} y_{i}, x_{i} y_{i+1}, x_{i} x_{i+1}, x_{i+1} y_{i}, y_{i} y_{i+1}, y_{i} z_{i}, y_{i} z_{i+1}, y_{i+1} z_{i}, z_{i} z_{i+1}\right\}, x_{n-2} y_{n-2}, y_{n-2} z_{n-2}\right)$ 
be a squarefree monomial ideal of $R:=K\left[x_{3}, \ldots, x_{n-2}, y_{3}, \ldots, y_{n-2}, z_{3}, \ldots, z_{n-2}\right]$. Then we have the following $K$-vector space isomorphism:

$$
\begin{aligned}
& I\left(\mathcal{C}_{n, 3}\right) / I\left(\mathcal{P}_{n, 3}\right) \cong \\
& y_{1} y_{n} \frac{R}{U}\left[y_{1}, y_{n}\right] \oplus x_{1} y_{n} \frac{R\left[z_{2}\right]}{\left(\mathcal{G}(U), y_{3} z_{2}, z_{2} z_{3}\right)}\left[x_{1}, y_{n}\right] \oplus z_{1} y_{n} \frac{R\left[x_{2}\right]}{\left(\mathcal{G}(U), y_{3} x_{2}, x_{2} x_{3}\right)}\left[z_{1}, y_{n}\right] \\
& \oplus y_{1} x_{n} \frac{R\left[z_{n-1}\right]}{\left(\mathcal{G}(U), y_{n-2} z_{n-1}, z_{n-2} z_{n-1}\right)}\left[y_{1}, x_{n}\right] \oplus y_{1} z_{n} \frac{R\left[x_{n-1}\right]}{\left(\mathcal{G}(U), y_{n-2} x_{n-1}, x_{n-2} x_{n-1}\right)}\left[y_{1}, z_{n}\right] \\
& \oplus x_{1} x_{n} \frac{R\left[z_{1}, z_{2}, z_{n-1}, z_{n}\right]}{\left(\mathcal{G}(U), y_{n-2} z_{n-1}, z_{n-2} z_{n-1}, z_{n-1} z_{n}, z_{n} z_{1}, z_{1} z_{2}, y_{3} z_{2}, z_{2} z_{3}\right)}\left[x_{1}, x_{n}\right] \\
& \oplus z_{1} z_{n} \frac{R\left[x_{1}, x_{2}, x_{n-1}, x_{n}\right]}{\left(\mathcal{G}(U), y_{n-2} x_{n-1}, x_{n-2} x_{n-1}, x_{n-1} x_{n}, x_{n} x_{1}, x_{1} x_{2}, y_{3} x_{2}, x_{2} x_{3}\right)}\left[z_{1}, z_{n}\right] .
\end{aligned}
$$

Clearly we can see that $R / U \cong S_{n-4,3} / I\left(\mathcal{P}_{n-4,3}\right)$,

$$
\begin{aligned}
\frac{R\left[z_{2}\right]}{\left(\mathcal{G}(U), y_{3} z_{2}, z_{2} z_{3}\right)} \cong \frac{R\left[x_{2}\right]}{\left(\mathcal{G}(U), y_{3} x_{2}, x_{2} x_{3}\right)} \cong \frac{R\left[z_{n-1}\right]}{\left(\mathcal{G}(U), y_{n-2} z_{n-1}, z_{n-2} z_{n-1}\right)} \\
\cong \frac{R\left[x_{n-1}\right]}{\left(\mathcal{G}(U), y_{n-2} x_{n-1}, x_{n-2} x_{n-1}\right)} \cong S_{n-4,3}^{\star} / I\left(\mathcal{P}_{n-4,3}^{\star}\right),
\end{aligned}
$$

and

$$
\begin{aligned}
& \frac{R\left[z_{1}, z_{2}, z_{n-1}, z_{n}\right]}{\left(\mathcal{G}(U), y_{n-2} z_{n-1}, z_{n-2} z_{n-1}, z_{n-1} z_{n}, z_{n} z_{1}, z_{1} z_{2}, y_{3} z_{2}, z_{2} z_{3}\right)} \\
& \cong \frac{R\left[x_{1}, x_{2}, x_{n-1}, x_{n}\right]}{\left(\mathcal{G}(U), y_{n-2} x_{n-1}, x_{n-2} x_{n-1}, x_{n-1} x_{n}, x_{n} x_{1}, x_{1} x_{2}, y_{3} x_{2}, x_{2} x_{3}\right)} \cong S_{n, 3}^{\diamond} / I\left(\mathfrak{C}_{n, 3}^{\diamond}\right) .
\end{aligned}
$$

Thus by Lemmas 3.2, 3.5, 4.5 and 2.7 we have

$$
\operatorname{sdepth}\left(I\left(\mathcal{C}_{n, 3}\right) / I\left(\mathcal{P}_{n, 3}\right)\right) \geq \min \left\{\left\lceil\frac{n-4}{3}\right\rceil+2,\left\lceil\frac{n-4+1}{3}\right\rceil+2,\left\lceil\frac{n-2}{3}\right\rceil+2\right\}=\left\lceil\frac{n+2}{3}\right\rceil .
$$

Theorem 4.7. For $1 \leq m \leq 3, n \geq 3$, $\operatorname{sdepth}\left(I\left(\mathcal{C}_{n, m}\right)\right) \geq \operatorname{sdepth}\left(S_{n, m} / I\left(\mathcal{C}_{n, m}\right)\right)$.

Proof. For $m=1, I\left(\mathfrak{C}_{n, 1}\right)=C_{n}$. Then the result follows by [4, Theorem 1.9] and [21, Theorem 2.3]. If $m=2$ or 3 , consider the short exact sequence

$$
0 \longrightarrow I\left(\mathcal{P}_{n, m}\right) \longrightarrow I\left(\mathcal{C}_{n, m}\right) \longrightarrow I\left(\mathcal{C}_{n, m}\right) / I\left(\mathcal{P}_{n, m}\right) \longrightarrow 0,
$$

then by Lemma 2.5, $\operatorname{sdepth}\left(I\left(\mathcal{C}_{n, m}\right)\right) \geq \min \left\{\operatorname{sdepth}\left(I\left(\mathcal{P}_{n, m}\right)\right), \operatorname{sdepth}\left(I\left(\mathcal{C}_{n, m}\right) / I\left(\mathcal{P}_{n, m}\right)\right)\right\}$. By Theorem 4.3 and we have $\operatorname{sdepth}\left(I\left(\mathcal{P}_{n, m}\right)\right) \geq\left\lceil\frac{n}{3}\right\rceil+1$, and by Propositions 4.4 and 4.6, we have $\operatorname{sdepth}\left(I\left(\mathcal{C}_{n, m}\right) / I\left(\mathcal{P}_{n, m}\right)\right) \geq\left\lceil\frac{n+2}{3}\right\rceil=\left\lceil\frac{n-1}{3}\right\rceil+1$, this completes the proof.

\section{Upper bounds for depth and Stanley depth of cyclic modules associ- ated to $\mathcal{P}_{n, m}$ and $\mathcal{C}_{n, m}$}

Let $m \leq n$, in general, we don't know the values of depth and Stanley depth of $S_{n, m} / I\left(\mathcal{P}_{n, m}\right)$. However, in the light of our observations, we propose the following question.

Question 1. Is $\operatorname{depth}\left(S_{n, m} / I\left(\mathcal{P}_{n, m}\right)\right)=\operatorname{sdepth}\left(S_{n, m} / I\left(\mathcal{P}_{n, m}\right)\right)=\left\lceil\frac{n}{3}\right\rceil\left\lceil\frac{m}{3}\right\rceil$ ?

Let $n \geq 2$, we have confirmed this question for the cases when $1 \leq m \leq 3$ see Remark 3.1, and Lemma 3.2. If $m=4$, we make some calculations for depth and Stanley depth by using CoCoA, (for sdepth we use SdepthLib:coc [25]). Calculations give an affirmative answer to Question 1 in the case $(n, m) \in\{(4,4),(5,4),(6,4)\}$. 
Theorem 5.1. For $n \geq 2, \operatorname{depth}\left(S_{n, m} / I\left(\mathcal{P}_{n, m}\right)\right), \operatorname{sdepth}\left(S_{n, m} / I\left(\mathcal{P}_{n, m}\right)\right) \leq\left\lceil\frac{n}{3}\right\rceil\left\lceil\frac{m}{3}\right\rceil$.

Proof. Without loss of generality, we assume that $m \leq n$. We first prove the result for depth. When $m=1$, then $I\left(\mathcal{P}_{n, 1}\right)=I\left(P_{n}\right)$, we have the required result by Remark 3.1. For $m=2,3$ the result follows from Lemma 3.2. Let $m \geq 4$, we will prove this result by induction on $m$. Let $v$ be a monomial such that

$$
v:= \begin{cases}x_{2(m-1)} x_{5(m-1)} \ldots x_{(n-4)(m-1)} x_{(n-1)(m-1)}, & \text { if } n \equiv 0(\bmod 3) \\ x_{1(m-1)} x_{4(m-1)} \ldots x_{(n-3)(m-1)} x_{n(m-1)}, & \text { if } n \equiv 1(\bmod 3) \\ x_{2(m-1)} x_{5(m-1)} \ldots x_{(n-3)(m-1)} x_{n(m-1)}, & \text { if } n \equiv 2(\bmod 3) .\end{cases}
$$

clearly $v \notin I\left(\mathcal{P}_{n, m}\right)$ so by Corollary 2.8

$$
\operatorname{depth}\left(S_{n, m} / I\left(\mathcal{P}_{n, m}\right)\right) \leq \operatorname{depth}\left(S_{n, m} /\left(I\left(\mathcal{P}_{n, m}\right): v\right)\right) .
$$

In all three cases $|\operatorname{supp}(v)|=\left\lceil\frac{n}{3}\right\rceil$ and $S_{n, m} /\left(I\left(\mathcal{P}_{n, m}\right): v\right) \cong\left(S_{n, m-3} / I\left(\mathcal{P}_{n, m-3}\right)\right)[\operatorname{supp}(v)]$, so by induction and Lemma 2.7

$$
\operatorname{depth}\left(S_{n, m} / I\left(\mathcal{P}_{n, m}\right)\right) \leq \operatorname{depth}\left(S_{n, m} /\left(I\left(\mathcal{P}_{n, m}\right): v\right)\right) \leq\left\lceil\frac{n}{3}\right\rceil\left\lceil\frac{m-3}{3}\right\rceil+\left\lceil\frac{n}{3}\right\rceil=\left\lceil\frac{m}{3}\right\rceil\left\lceil\frac{n}{3}\right\rceil .
$$

Similarly, we can prove the result for Stanley depth by using Proposition 2.9.

Remark 5.2. For a positive answer to Question 1, one needs to prove that $\left\lceil\frac{n}{3}\right\rceil\left\lceil\frac{m}{3}\right\rceil$ is a lower bound for depth and Stanley depth of $S_{n, m} / I\left(\mathcal{P}_{n, m}\right)$. The lower bound $\left\lceil\frac{\operatorname{diam}\left(P_{n, m}\right)+1}{3}\right\rceil$ from Corollaries 2.11 and 2.13 which was helpful for the cases when $1 \leq m \leq 3$ is no more useful if $m \geq 4$. For instance, $\operatorname{depth}\left(S_{4,4} / I\left(\mathcal{P}_{4,4}\right)\right)=\operatorname{sdepth}\left(S_{4,4} / I\left(\mathcal{P}_{4,4}\right)\right)=$ 4 , but this lower bound shows that $\operatorname{depth}\left(S_{4,4} / I\left(\mathcal{P}_{4,4}\right)\right) \geq 2=\left\lceil\frac{\operatorname{diam}\left(P_{4,4}\right)+1}{3}\right\rceil$ and $\operatorname{sdepth}\left(S_{4,4} / I\left(\mathcal{P}_{4,4}\right)\right) \geq 2=\left\lceil\frac{\operatorname{diam}\left(P_{4,4}\right)+1}{3}\right\rceil$.

Theorem 5.3. For $n \geq 3$ and $m \geq 1$,

$$
\operatorname{depth}\left(S_{n, m} / I\left(\mathfrak{C}_{n, m}\right)\right) \leq \begin{cases}\left\lceil\frac{n-1}{3}\right\rceil+\left(\left\lceil\frac{m}{3}\right\rceil-1\right)\left\lceil\frac{n}{3}\right\rceil, & \text { if } m \equiv 1,2(\bmod 3) ; \\ \left\lceil\frac{n}{3}\right\rceil\left\lceil\frac{m}{3}\right\rceil, & \text { if } m \equiv 0(\bmod 3) .\end{cases}
$$

Proof. We prove this result by induction on $m$. If $m=1$, then $I\left(\mathfrak{C}_{n, 1}\right)=I\left(C_{n}\right)$, by [4, Proposition 1.3], we have the required result. For $m=2,3$ the result follows by Theorems 3.3 and 3.6, respectively. Let $m \geq 4$,

$$
u:= \begin{cases}x_{3(m-1)} x_{6(m-1)} \ldots x_{(n-3)(m-1)} x_{n(m-1)}, & \text { if } n \equiv 0(\bmod 3) ; \\ x_{1(m-1)} x_{4(m-1)} \ldots x_{(n-6)(m-1)} x_{(n-3)(m-1)} x_{(n-1)(m-1)}, & \text { if } n \equiv 1(\bmod 3) ; \\ x_{2(m-1)} x_{5(m-1)} \ldots x_{(n-3)(m-1)} x_{n(m-1)}, & \text { if } n \equiv 2(\bmod 3) .\end{cases}
$$

Clearly $u \notin I\left(\mathcal{C}_{n, m}\right)$ and $S_{n, m} /\left(I\left(\mathcal{C}_{n, m}\right): u\right) \cong\left(S_{n, m-3} / I\left(\mathcal{C}_{n, m-3}\right)\right)[\operatorname{supp}(u)]$, since in all the cases $|\operatorname{supp}(u)|=\left\lceil\frac{n}{3}\right\rceil$, if $m \equiv 1,2(\bmod 3)$ so by induction and Lemma 2.7

$\operatorname{depth}\left(S_{n, m} /\left(I\left(\mathcal{C}_{n, m}\right): u\right)\right) \leq\left\lceil\frac{n-1}{3}\right\rceil+\left(\left\lceil\frac{m-3}{3}\right\rceil-1\right)\left\lceil\frac{n}{3}\right\rceil+\left\lceil\frac{n}{3}\right\rceil=\left\lceil\frac{n-1}{3}\right\rceil+\left(\left\lceil\frac{m}{3}\right\rceil-1\right)\left\lceil\frac{n}{3}\right\rceil$.

Otherwise, by induction and Lemma 2.7 we have

$$
\operatorname{depth}\left(S_{n, m} /\left(I\left(\mathfrak{C}_{n, m}\right): u\right)\right) \leq\left\lceil\frac{n}{3}\right\rceil\left\lceil\frac{m-3}{3}\right\rceil+\left\lceil\frac{n}{3}\right\rceil=\left\lceil\frac{n}{3}\right\rceil\left\lceil\frac{m}{3}\right\rceil .
$$

Theorem 5.4. For $n \geq 3$ and $m \geq 1, \operatorname{sdepth}\left(S_{n, m} / I\left(\mathrm{C}_{n, m}\right)\right) \leq\left\lceil\frac{n}{3}\right\rceil\left\lceil\frac{m}{3}\right\rceil$.

Proof. The proof is similar to the proof of Theorem 5.3 by using Corollary 3.4 instead of Theorems 3.3.

Remark 5.5. The upper bounds for Stanley depth of $S_{n, m} / I\left(\mathcal{P}_{n, m}\right)$ and $S_{n, m} / I\left(\mathcal{C}_{n, m}\right)$ as proved in Theorems 5.1 and 5.4 are too sharp. On the bases of our observations, we formulate the following question. A positive answer to this question will prove Conjecture 1. 
Question 2. Is $\operatorname{sdepth}\left(I\left(\mathcal{P}_{n, m}\right)\right), \operatorname{sdepth}\left(I\left(\mathcal{C}_{n, m}\right)\right) \geq\left\lceil\frac{n}{3}\right\rceil\left\lceil\frac{m}{3}\right\rceil ?$

\section{References}

[1] C. Bir, D.M. Howard, M.T. Keller, W.T. Trotter and S.J. Young, Interval partitions and Stanley depth, J. Combin. Theory Ser. A, 117, 475-482, 2010.

[2] M. Cimpoeaş, Several inequalities regarding Stanley depth, Romanian Journal of Math. and Computer Science, 2, 28-40, 2012.

[3] M. Cimpoeaş, Stanley depth of squarefree Veronese ideals, An. St. Univ. Ovidius Constanta, 21 (3), 67-71, 2013.

[4] M. Cimpoeaş, On the Stanley depth of edge ideals of line and cyclic graphs, Romanian Journal of Math. and Computer Science, 5 (1), 70-75, 2015.

[5] CoCoATeam, CoCoA: a system for doing Computations in Commutative Algebra, available at http://cocoa.dima.unige.it.

[6] A.M. Duval, B. Goeckner, C.J. Klivans and J.L. Martine, A non-partitionable CohenMacaulay simplicial complex, Adv. Math. 299, 381-395, 2016.

[7] S.A.S. Fakhari, On the Stanley Depth of Powers of Monomial Ideals, Mathematics, 7, 607, 2019.

[8] L. Fouli and S. Morey, A lower bound for depths of powers of edge ideals, J. Algebraic Combin. 42 (3), 829-848, 2015.

[9] R. Hammack, W. Imrich and S. Klavar, Handbook of Product Graphs, Second Edition, CRC Press, Boca Raton, FL, 2011.

[10] J. Herzog, A survey on Stanley depth, In Monomial ideals, computations and applications, Lecture Notes in Math. 2083, Springer, Heidelberg, 3-45, 2013.

[11] J. Herzog, M. Vladoiu and X. Zheng, How to compute the Stanley depth of a monomial ideal, J. Algebra, 322 (9), 3151-3169, 2009.

[12] Z. Iqbal and M. Ishaq, Depth and Stanley depth of edge ideals associated to some line graphs, AIMS Mathematics, 4 (3), 686-698, 2019.

[13] Z. Iqbal and M. Ishaq, Depth and Stanley depth of edge ideals of powers of paths and cycles, An. Şt. Univ. Ovidius Constana, 27 (3), 113-135, 2019.

[14] Z. Iqbal, M. Ishaq and M. Aamir, Depth and Stanley depth of edge ideals of square paths and square cycles, Comm. Algebra, 46 (3), 1188-1198, 2018.

[15] M. Ishaq, Upper bounds for the Stanley depth, Comm. Algebra, 40 (1), 87-97, 2012.

[16] M. Ishaq, Values and bounds for the Stanley depth, Carpathian J. Math. 27 (2), 217-224, 2011.

[17] M. Ishaq and M.I. Qureshi, Upper and lower bounds for the Stanley depth of certain classes of monomial ideals and their residue class rings, Comm. Algebra, 41 (3), 1107-1116, 2013.

[18] M.T. Keller and S.J. Young, Combinatorial reductions for the Stanley depth of I and S/I, Electron. J. Comb. 24 (3), \#P3.48, 2017.

[19] M.T. Keller, Y. Shen, N. Streib and S.J. Young, On the Stanley depth of squarefree veronese ideals, J. Algebraic Combin. 33 (2), 313-324, 2011.

[20] S. Morey, Depths of powers of the edge ideal of a tree, Comm. Algebra, 38 (11), 4042-4055, 2010.

[21] R. Okazaki, A lower bound of Stanley depth of monomial ideals, J. Commut. Algebra, 3 (1), 83-88, 2011.

[22] M.R. Pournaki, S.A.S. Fakhari and S. Yassemi, Stanley depth of powers of the edge ideals of a forest, Proc. Amer. Math. Soc. 141 (10), 3327-3336, 2013.

[23] M.R. Pournaki, S.A.S. Fakhari, M. Tousi and S. Yassemi, What is ... Stanley depth? Not. Am. Math. Soc. 56, 1106-1108, 2009.

[24] A. Rauf, Depth and Stanley depth of multigraded modules, Comm. Algebra, 38 (2), 773-784, 2010. 
[25] G. Rinaldo, An algorithm to compute the Stanley depth of monomial ideals, Le Matematiche, LXIII(ii), 243-256, 2008.

[26] R.P. Stanley, Linear Diophantine equations and local cohomology, Invent. Math. 68 (2), 175-193, 1982.

[27] A. Stefan, Stanley depth of powers of path ideal, http://arxiv.org/pdf/1409.6072.pdf.

[28] R.H. Villarreal, Monomial Algebras in:Monographs and Textbooks in Pure and Applied Mathematics, Marcel Dekker, Inc., New York, 238, 2011. 